\title{
A Geometrical Three-Ring-Based Model for MIMO Mobile-to-Mobile Fading Channels in Cooperative Networks
}

\author{
Batool Talha and Matthias Pätzold \\ Faculty of Engineering and Science, University of Agder, Servicebox 509, 4898 Grimstad, Norway \\ Correspondence should be addressed to Batool Talha, batool.talha@uia.no
}

Received 2 June 2010; Revised 4 October 2010; Accepted 2 January 2011

Academic Editor: Francesco Verde

Copyright (C) 2011 B. Talha and M. Pätzold. This is an open access article distributed under the Creative Commons Attribution License, which permits unrestricted use, distribution, and reproduction in any medium, provided the original work is properly cited.

This paper deals with the modeling and analysis of narrowband multiple-input multiple-output (MIMO) mobile-to-mobile (M2M) fading channels in relay-based cooperative networks. In the transmission links from the source mobile station to the destination mobile station via the mobile relay, non-line-of-sight (NLOS) propagation conditions are taken into account. A stochastic narrowband MIMO M2M reference channel model is derived from the geometrical three-ring scattering model, where it is assumed that an infinite number of local scatterers surround the source mobile station, the mobile relay, and the destination mobile station. The complex channel gains associated with the new reference channel model are derived, and their temporal as well as spatial correlation properties are explored. General analytical solutions are obtained for the four-dimensional (4D) space-time cross-correlation function (CCF), the three-dimensional (3D) spatial CCF, the two-dimensional (2D) source (relay, destination) correlation function (CF), and the temporal autocorrelation function (ACF). Exact closed-form expressions for different CFs under isotropic as well as nonisotropic scattering conditions are provided in this article. A stochastic simulation model is then drawn from the reference model. It is shown that the CCFs of the simulation model closely approximate the corresponding CCFs of the reference model. The developed channel simulator is not only important for the development of future MIMO M2M cooperative communication systems, but also for analyzing the dynamic behavior of the MIMO M2M channel capacity.

\section{Introduction}

Recent attempts to combat multipath fading effects along with providing increased mobility support have resulted in the emergence of M2M communication systems in cooperative networks. The use of cooperative diversity protocols [1-4] improves the transmission link quality and the endto-end system throughput, whereas M2M communication, on the other hand, expands the network range (coverage area). The fundamental idea of cooperative networks is to allow mobile stations in the network to relay signals to the final destination or to other mobile stations acting as relays. The development and performance investigation of such seemingly straightforward cooperative networks require a thorough understanding of the $\mathrm{M} 2 \mathrm{M}$ fading channel characteristics. For this reason, there is a need for simple yet efficient M2M fading channel models, providing us with a detailed knowledge about the statistical characterization of M2M channels.

The idea of introducing M2M communication in noncooperative networks can be traced back to the work of Akki and Haber $[5,6]$, which deals with the study of the statistical properties of narrowband single-input single-output (SISO) M2M fading channels under non-line-of-sight (NLOS) propagation conditions. Several papers dealing with M2M communication in cooperative networks can be found in the recent literature [7-9]. In various studies regarding M2M fading channels in relay-based cooperative networks under NLOS propagation conditions, it has been shown that a double Rayleigh process is an unsophisticated but still a well-suited statistical channel model for such channels [10, 11]. Besides, the credit of reporting the temporal ACF of 
fading channels in amplify-and-forward relay systems goes to Patel et al. [11]. The analysis of experimental measurement data for outdoor-to-indoor M2M fading channels included in [12] verifies the existence of double Rayleigh processes in real-world environments. Talha and Pätzold [7] have extended the double Rayleigh channel model to the double Rice channel model for line-of-sight (LOS) propagation environments. Furthermore, a variety of other realistic M2M fading channel models based on the multiple scattering concept [13] are available in the literature for both NLOS and LOS propagation environments $[9,14]$. The M2M fading channel models for relay-based cooperative networks proposed to date are for narrowband SISO fading channels. Meaning thereby, the source mobile station, the mobile relay, and the destination mobile station are equipped with only one antenna. However, it is a well-established fact that the gains in terms of channel capacity are larger for MIMO channels as compared to SISO channels $[15,16]$. This thus calls for an extension of SISO M2M channel models to MIMO M2M channel models, since such models facilitate investigations pertaining to the channel capacity and the system performance of cooperative networks with multiple antenna models.

Another area that requires further attention is the development of simulation models for MIMO M2M fading channels in cooperative networks. Some techniques for simulating narrowband SISO M2M fading channels in noncooperative networks can be found in [17]. Various studies have revealed that geometrical channel models are a good starting point for deriving simulation models for MIMO channels. Quite a lot of narrowband MIMO channel models based on geometrical scattering models for isotropic environments have been developed so far [18-25]. The design of geometry-based MIMO channel models for nonisotropic scattering conditions is addressed in [26, 27], whereas wideband MIMO channel models are discussed in $[28,29]$. The common feature in the works [18-29] is that they model MIMO fixed-to-mobile (F2M) and/or fixed-to-fixed (F2F) channels. The geometrical two-ringbased model for MIMO F2M channels originally proposed in [21] was extended to a narrowband MIMO M2M channel model by Pätzold et al. [30]. Zajić and Stüber [31] have reported on MIMO M2M reference and simulation models under LOS propagation conditions. The geometrical street model [32] and the geometrical T-junction model [33] for MIMO M2M fading channels are worth mentioning. All the geometry-based channel models mentioned to this point in the current section are $2 \mathrm{D}$ channel models. There are also Zajić and Stüber [34] who have successfully expanded 2D MIMO M2M channel models to 3D models based on geometrical cylinders. Nonetheless, to the best of the authors' knowledge, geometrical channel models for MIMO M2M communication systems in cooperative networks are an unexplored area. This in turn results in a lack of proper reference and simulation models derived from such geometrical models for MIMO M2M fading channels.

Motivated by the need for proper MIMO M2M fading channel models, we are addressing in this article modeling and simulation approaches for such channels in amplifyand-forward relay-type cooperative networks. Additionally, there was not a single M2M channel model for cooperative networks available in the literature, which assumes multiple antennas on the source mobile station, the destination mobile station, or the mobile relays. This gap in the research propelled us to introduce a geometry-based model for MIMO M2M channels in relay-based systems. The scattering environment around the source mobile station, the mobile relay, and the destination mobile station are modeled by a geometrical three-ring scattering model. The advanced geometrical three-ring scattering model is an extension of the geometrical two-ring scattering model presented in [30], where the source mobile station and the destination mobile station are surrounded by rings of scatterers. However, in the suggested extension of the two-ring model to the three-ring model, we have a separate ring of scatterers around the mobile relay in addition to a ring around each source mobile station and destination mobile station. For simplicity, the reference model introduced in this article caters for MIMO M2M fading channels under NLOS propagation conditions only. Moreover, it is assumed that the direct transmission link between the source mobile station and the destination mobile station is blocked by obstacles. Since geometrybased MIMO channel models are usually characterized by their temporal as well as spatial correlation properties, we explore the correlation properties of our devised threering-based model here. It is noteworthy that while deriving the temporal ACF of the relay links, the authors of [11] took into consideration such propagation scenarios where a stationary base station (BS) acts either as a source (i.e., transmitter) or a relay. It is usually supposed that the BS is elevated and unobstructed. It is further believed that the BS is not surrounded by local scatterers. This assumption of the elevated BS makes [11] different from our work. Additionally, we derive a stochastic simulation model from the developed reference model. Finally, we discuss the nonisotropic scattering scenario along with the isotropic one as a special case and present closed-form expressions for the correlation functions of the reference as well as simulation models.

This article has the following structure. Section 2 introduces briefly the geometrical three-ring scattering model describing the transmission link from the source mobile station to the destination mobile station via the mobile relay. Based on the geometrical three-ring scattering model, we develop the reference model for MIMO M2M fading channels and study its correlation properties in Section 3. In Section 6, we derive the stochastic simulation model from the developed reference model. Section 4 deals with the derivation of closed-form expressions for the correlation functions describing the reference model under nonisotropic scattering conditions. Section 5 shows the accuracy of the stochastic simulation model by comparing its statistical properties with those of the reference model. In this section, we also confirm the validity of the closed-form expressions obtained in Section 4. Finally, concluding remarks are given in Section 7. 


\section{The Geometrical Three-Ring Model}

In this section, we extend the geometrical two-ring scattering model proposed in [30] to a geometrical three-ring scattering model for narrowband MIMO M2M fading channels in amplify-and-forward relay-type cooperative networks. For ease of analysis, we have considered an elementary $2 \times 2 \times 2$ antenna configuration, meaning thereby, the source mobile station, the mobile relay, and the destination mobile station are equipped with two antennas each. For simplicity, NLOS propagation conditions have been taken into account in all the transmission links. It is further assumed that there is no direct transmission link from the source mobile station to the destination mobile station.

Due to high path loss, the contribution of signal power from remote scatterers to the total received power is usually negligible. In this context, the recommended three-ring scattering model only accommodates local scattering. A total number of $M$ local scatterers, that is, $S_{S}^{(m)}(m=$ $1,2, \ldots, M)$ are positioned on a ring of radius $R_{\mathrm{S}}$ around the source mobile station, whereas $N$ local scatterers $S_{\mathrm{D}}^{(n)}(n=$ $1,2, \ldots, N)$ lie around the destination mobile station on a separate ring of radius $R_{\mathrm{D}}$. Besides, the local scatterers $S_{\mathrm{R}}^{(k)}(k=1,2, \ldots, K)$ and $S_{\mathrm{R}}^{(l)}(l=1,2, \ldots, L)$ are located on a third ring of radius $R_{\mathrm{R}}$ around the mobile relay. The number of local scatterers around the mobile relay is $K=L$. It should be pointed out here that $S_{\mathrm{R}}^{(k)}=S_{\mathrm{R}}^{(l)}$ for $k=l$. Throughout this paper, the subscripts $S, R$, and $D$ represent the source mobile station, the mobile relay, and the destination mobile station, respectively. As can be seen from Figure 1, the symbol $\phi_{S}^{(m)}$ denotes the angle of departure (AOD) of the $m$ th transmitted wave seen at the source mobile station, whereas $\phi_{\mathrm{D}}^{(n)}$ represents the angle of arrival (AOA) of the $n$th received wave at the destination mobile station. Furthermore, the symbols $\phi_{\mathrm{S}-\mathrm{R}}^{(k)}$ and $\phi_{\mathrm{R}-\mathrm{D}}^{(l)}$ correspond to the AOA of the $k$ th received wave and the AOD of the $l$ th transmitted wave at the mobile relay, respectively. The mobile relay is positioned at a distance $D_{S-R}$ and an angle $\gamma_{S}$ with respect to the source mobile station. While the location of the mobile relay seen from the destination mobile station can be specified by the distance $D_{\mathrm{R}-\mathrm{D}}$ and the angle $\gamma_{\mathrm{D}}$. In addition, the source mobile station and the destination mobile station are a distance $D_{S-D}$ apart from each other. It is expected that the inequalities $\max \left\{R_{\mathrm{S}}, R_{\mathrm{R}}\right\} \ll D_{\mathrm{S}-\mathrm{R}}, \max \left\{R_{\mathrm{R}}, R_{\mathrm{D}}\right\} \ll$ $D_{\text {R-D }}$, and $\max \left\{R_{\mathrm{S}}, R_{\mathrm{D}}\right\} \ll D_{\mathrm{S}-\mathrm{D}}$ hold. The interelement spacings at the source mobile station, the mobile relay, and the destination mobile station antenna arrays are labeled as $\delta_{S}, \delta_{R}$, and $\delta_{D}$, respectively, where it is understood that these quantities are smaller than the radii $R_{\mathrm{S}}, R_{\mathrm{R}}$, and $R_{\mathrm{D}}$, that is, $\max \left\{\delta_{\mathrm{S}}, \delta_{\mathrm{R}}, \delta_{\mathrm{D}}\right\} \ll \min \left\{R_{\mathrm{S}}, R_{\mathrm{R}}, R_{\mathrm{D}}\right\}$. With respect to the $x$-axis, the symbols $\beta_{\mathrm{S}}, \beta_{\mathrm{R}}$, and $\beta_{\mathrm{D}}$ describe the tilt angle of the antenna arrays at the source mobile station, the mobile relay, and the destination mobile station, respectively. Additionally, it is supposed that the source mobile station (mobile relay, destination mobile station) moves with speed $\mathrm{v}_{\mathrm{S}}\left(\mathrm{v}_{\mathrm{R}}, \mathrm{v}_{\mathrm{D}}\right)$ in the direction determined by the angle of motion $\alpha_{\mathrm{S}}\left(\alpha_{\mathrm{R}}, \alpha_{\mathrm{D}}\right)$.

\section{The Reference Model}

3.1. Derivation of the Reference Model. In this section, we develop a reference model for MIMO M2M fading channels in cooperative networks using the geometrical threering scattering model shown in Figure 1. Ignoring for the moment the geometrical details, Figure 1 can be simplified to Figure 2, in order to understand the overall MIMO channel from the source mobile station to the destination mobile station via the mobile relay. Figure 2 shows that the complete system can be separated into two $2 \times 2$ MIMO subsystems. One of the MIMO subsystems (comprising the source mobile station and mobile relay) is denoted by the S-R MIMO subsystem. While the other MIMO subsystem (consisting of the mobile relay and the destination mobile station) is termed as the R-D MIMO subsystem. The input-output relationship of the S-R MIMO subsystem can be expressed as

$$
\mathbf{X}(t)=\mathbf{H}_{\mathrm{S}-\mathrm{R}}(t) \mathbf{S}(t)+\mathbf{N}_{\mathrm{R}}(t),
$$

where $\mathbf{X}(t)=\left[\begin{array}{ll}X^{(1)}(t) & X^{(2)}(t)\end{array}\right]^{T}$ is a $2 \times 1$ received signal vector at the mobile relay, $\mathbf{S}(t)=\left[S^{(1)}(t) S^{(2)}(t)\right]^{T}$ is a $2 \times 1$ signal vector transmitted by the source mobile station, and $\mathbf{N}_{\mathrm{R}}(t)=\left[\begin{array}{lll}N_{\mathrm{R}}^{(1)}(t) & N_{\mathrm{R}}^{(2)}(t)\end{array}\right]^{T}$ is a $2 \times 1$ additive white Gaussian noise (AWGN) vector. In (1), $\mathbf{H}_{\mathrm{S}-\mathrm{R}}(t)$ is a $2 \times 2$ channel matrix, which models the M2M fading channel between the source mobile station and the mobile relay. The channel matrix $\mathbf{H}_{\mathrm{S}-\mathrm{R}}(t)$ can be expressed as

$$
\mathbf{H}_{\mathrm{S}-\mathrm{R}}(t)=\left(\begin{array}{cc}
h_{\mathrm{S}-\mathrm{R}}^{(11)}(t) & h_{\mathrm{S}-\mathrm{R}}^{(12)}(t) \\
h_{\mathrm{S}-\mathrm{R}}^{(21)}(t) & h_{\mathrm{S}-\mathrm{R}}^{(22)}(t)
\end{array}\right) .
$$

Here, each element $h_{\mathrm{S}-\mathrm{R}}^{(i q)}(t)(i, q=1,2)$ of the channel matrix represents the diffuse component of the channel describing the transmission link from the source mobile station antenna element $A_{\mathrm{S}}^{(q)}$ to the mobile relay antenna element $A_{\mathrm{R}}^{(i)}$. Considering the geometrical three-ring scattering model shown in Figure 1, it can be observed that the $m$ th homogeneous plane wave emitted from $A_{\mathrm{S}}^{(q)}$, first encounters the local scatterers $S_{\mathrm{S}}^{(m)}$ around the source mobile station. Moreover, before impinging on $A_{\mathrm{R}}^{(i)}$, the plane wave is captured by the local scatterers $S_{\mathrm{R}}^{(k)}$ around the mobile relay. It is worth mentioning here that the reference model is based on the assumption that the number of local scatterers, $M$ and $K$, around the source mobile station and the mobile relay is infinite. Following [30], the diffuse component $h_{\mathrm{S}-\mathrm{R}}^{(11)}(t)$ of the transmission link from $A_{\mathrm{S}}^{(1)}$ to $A_{\mathrm{R}}^{(1)}$ can be approximated as

$$
h_{\mathrm{S}-\mathrm{R}}^{(11)}(t)=\lim _{\substack{M \rightarrow \infty \\ K \rightarrow \infty}} \frac{1}{\sqrt{M K}} \sum_{m=1}^{M} \sum_{k=1}^{K} g_{S-\mathrm{R}}^{(m k)} e^{j\left[2 \pi\left(f_{\mathrm{S}}^{(m)}+f_{\mathrm{S}-\mathrm{R}}^{(k)}\right) t+\left(\theta_{\mathrm{S}-\mathrm{R}}^{(m)}+\theta_{\mathrm{S}-\mathrm{R}}\right)\right]}
$$

with joint gains $1 / \sqrt{M K}$ and joint phases $\theta_{S-R}^{(m k)}$ caused by the interaction of the local scatterers $S_{\mathrm{S}}^{(m)}$ and $S_{\mathrm{R}}^{(k)}$. The joint phases $\theta_{\mathrm{S}-\mathrm{R}}^{(m k)}$ are considered to be independent and identically 


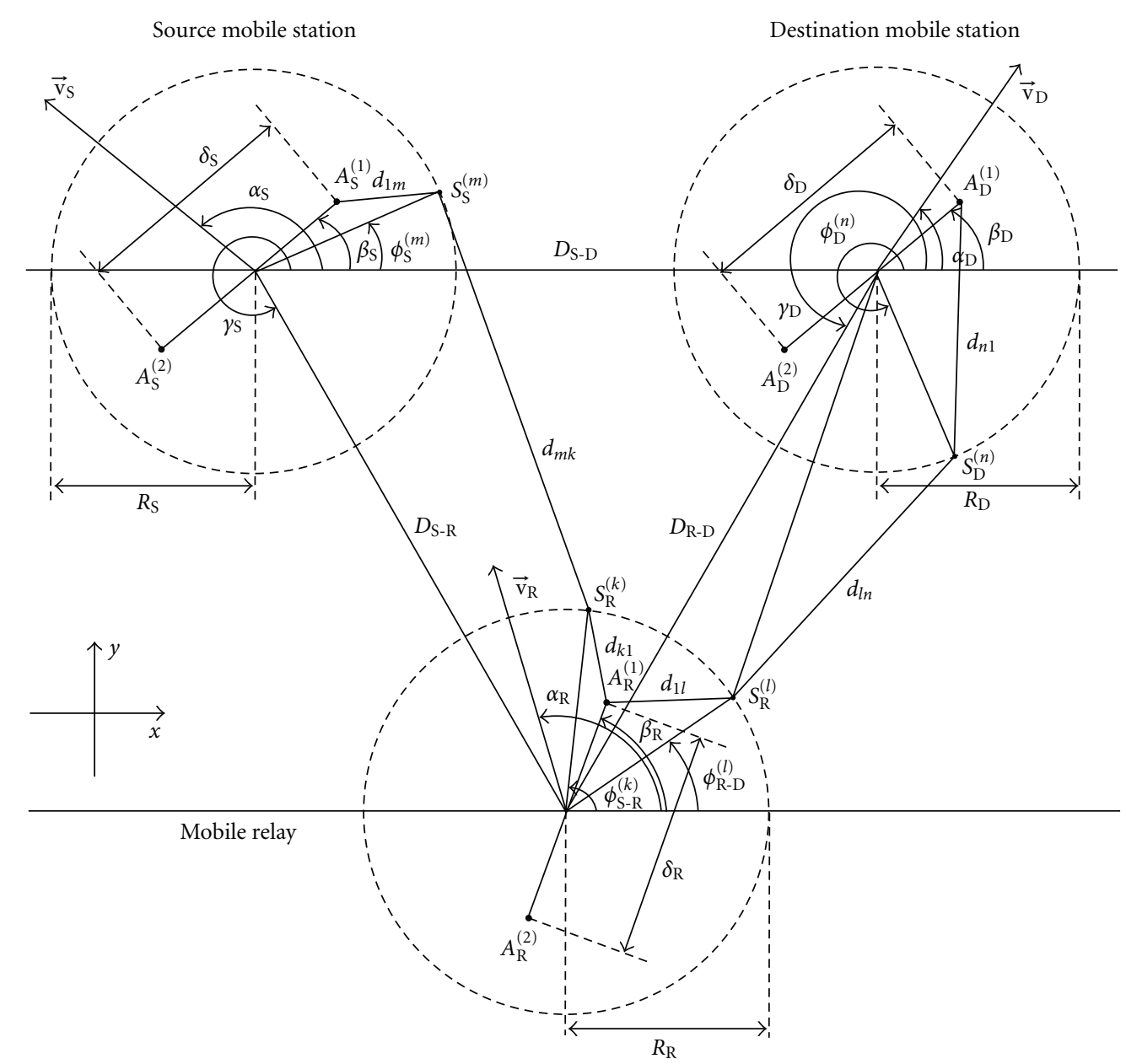

FIGURE 1: The geometrical three-ring scattering model for a $2 \times 2 \times 2 \mathrm{MIMO}$ M2M channel with local scatterers on rings around the source mobile station, the mobile relay, and the destination mobile station.

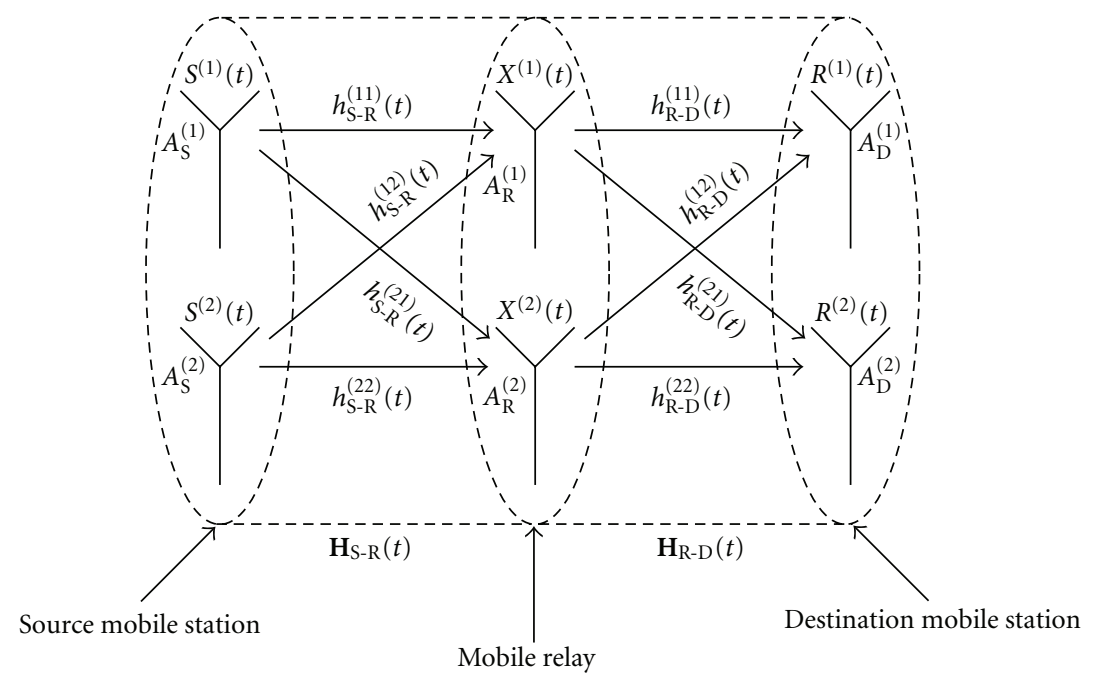

FIGURE 2: A simplified diagram describing the overall MIMO M2M channel from the source mobile station to the destination mobile station via the mobile relay. 
distributed (i.i.d.) random variables, each having a uniform distribution over the interval $[0,2 \pi]$. In (3),

$$
\begin{aligned}
g_{\mathrm{S}-\mathrm{R}}^{(m k)} & =a_{\mathrm{S}}^{(m)} b_{\mathrm{R}}^{(k)} c_{\mathrm{S}-\mathrm{R}}^{(m k)}, \\
a_{\mathrm{S}}^{(m)} & =e^{j(\pi / \lambda) \delta_{\mathrm{S}} \cos \left(\phi_{\mathrm{S}}^{(m)}-\beta_{\mathrm{S}}\right)}, \\
b_{\mathrm{R}}^{(k)} & =e^{j(\pi / \lambda) \delta_{\mathrm{R}} \cos \left(\phi_{\mathrm{S}-\mathrm{R}}^{(k)}-\beta_{\mathrm{R}}\right)}, \\
c_{\mathrm{S}-\mathrm{R}}^{(m k)} & =e^{j(2 \pi / \lambda)\left\{R_{\mathrm{S}} \cos \left(\phi_{\mathrm{S}}^{(m)}-\gamma_{\mathrm{S}}\right)-R_{\mathrm{R}} \cos \left(\phi_{\mathrm{S}-\mathrm{R}}^{(k)}-\gamma_{\mathrm{S}}\right)\right\}}, \\
\theta_{\mathrm{S}-\mathrm{R}} & =-\frac{2 \pi}{\lambda}\left(R_{\mathrm{S}}+D_{\mathrm{S}-\mathrm{R}}+R_{\mathrm{R}}\right), \\
f_{\mathrm{S}}^{(m)} & =f_{\mathrm{S}_{\max }} \cos \left(\phi_{\mathrm{S}}^{(m)}-\alpha_{\mathrm{S}}\right), \\
f_{\mathrm{S}-\mathrm{R}}^{(k)} & =f_{\mathrm{R}_{\max }} \cos \left(\phi_{\mathrm{S}-\mathrm{R}}^{(k)}-\alpha_{\mathrm{R}}\right),
\end{aligned}
$$

where $f_{\mathrm{S}_{\max }}=\mathrm{v}_{\mathrm{S}} / \lambda\left(f_{\mathrm{R}_{\max }}=\mathrm{v}_{\mathrm{R}} / \lambda\right)$ is the maximum Doppler frequency caused by the motion of the source mobile station (mobile relay) and $\lambda$ denotes the carrier's wavelength. The knowledge of the position of the mobile relay with respect to the source mobile station is incorporated in (4d). It should be pointed out here that in the reference model, the AOD $\phi_{\mathrm{S}}^{(m)}$, and the AOA $\phi_{\mathrm{S}-\mathrm{R}}^{(k)}$ are independent random variables determined by the distribution of the local scatterers around the source mobile station and the mobile relay, respectively.

Replacing $a_{\mathrm{S}}^{(m)}$ and $b_{\mathrm{R}}^{(k)}$ by their complex conjugates $a_{\mathrm{S}}^{(m) *}$ and $b_{\mathrm{R}}^{(k) *}$ in $(4 \mathrm{~b})$ and $(4 \mathrm{c})$, respectively, we can obtain the diffuse component $h_{\mathrm{S}-\mathrm{R}}^{(22)}(t)$ of the $A_{\mathrm{S}}^{(2)}-A_{\mathrm{R}}^{(2)}$ transmission link [30]. The diffuse components $h_{\mathrm{S}-\mathrm{R}}^{(12)}(t)$ and $h_{\mathrm{S}-\mathrm{R}}^{(21)}(t)$ can be realized likewise by substituting $a_{\mathrm{S}}^{(m)} \rightarrow a_{\mathrm{S}}^{(m) *}$ and $b_{\mathrm{R}}^{(k)} \rightarrow$ $b_{\mathrm{R}}^{(k) *}$, respectively, in (3) [30].

In the same way, it is evident from Figure 2 that the input-output relationship of the R-D MIMO subsystem can be written as

$$
\mathbf{R}(t)=\mathbf{H}_{\mathrm{R}-\mathrm{D}}(t) \mathbf{X}(t)+\mathbf{N}_{\mathrm{D}}(t)
$$

where $\mathbf{R}(t)=\left[R^{(1)}(t) R^{(2)}(t)\right]^{T}$ is a $2 \times 1$ received signal vector at the destination mobile station, $\mathbf{X}(t)=\left[X^{(1)}(t) X^{(2)}(t)\right]^{T}$ is a $2 \times 1$ signal vector transmitted by the mobile relay, $\mathbf{H}_{\mathrm{R}-\mathrm{D}}(t)$ is a $2 \times 2 \mathrm{R}-\mathrm{D}$ fading channel matrix, and $\mathrm{N}_{\mathrm{D}}(t)=\left[N_{\mathrm{D}}^{(1)}(t) N_{\mathrm{D}}^{(2)}(t)\right]^{T}$ is a $2 \times 1$ AWGN vector. By referring to the previous discussion on the elements of the matrix $\mathbf{H}_{\mathrm{S}-\mathrm{R}}(t)$, one can easily show that the diffuse component $h_{\mathrm{R}-\mathrm{D}}^{(11)}(t)$ of the $A_{\mathrm{R}}^{(1)}-A_{\mathrm{D}}^{(1)}$ transmission link can be expressed as

$$
h_{\mathrm{R}-\mathrm{D}}^{(11)}(t)=\lim _{\substack{L \rightarrow \infty \\ N \rightarrow \infty}} \frac{1}{\sqrt{L N}} \sum_{l=1}^{L} \sum_{n=1}^{N} g_{\mathrm{R}-\mathrm{D}}^{(l n)} e^{j\left[2 \pi\left(f_{\mathrm{R}-\mathrm{D}}^{(l)}+f_{\mathrm{D}}^{(n)}\right) t+\left(\theta_{\mathrm{R}-\mathrm{D}}^{(l n)}+\theta_{\mathrm{R}-\mathrm{D}}\right)\right]},
$$

where the term $1 / \sqrt{L N}$ and the symbol $\theta_{\mathrm{R}-\mathrm{D}}^{(\text {ln })}$ represent the joint gains and joint phases, respectively, introduced by the local scatterers $S_{\mathrm{R}}^{(l)}$ and $S_{\mathrm{D}}^{(n)}$. Like the joint phases, $\theta_{\mathrm{S}-\mathrm{R}}^{(m k)}$, $\theta_{\mathrm{R}-\mathrm{D}}^{(l n)}$ are assumed to be i.i.d. random variables as well, each having a uniform distribution over the interval $[0,2 \pi]$. Furthermore, in (6),

$$
\begin{aligned}
g_{\mathrm{R}-\mathrm{D}}^{(l n)} & =a_{\mathrm{R}}^{(l)} b_{\mathrm{D}}^{(n)} c_{\mathrm{R}-\mathrm{D}}^{(l n)}, \\
a_{\mathrm{R}}^{(l)} & =e^{j(\pi / \lambda) \delta_{\mathrm{R}} \cos \left(\phi_{\mathrm{R}-\mathrm{D}}^{(l)}-\beta_{\mathrm{R}}\right)}, \\
b_{\mathrm{D}}^{(n)} & =e^{j(\pi / \lambda) \delta_{\mathrm{D}} \cos \left(\phi_{\mathrm{D}}^{(n)}-\beta_{\mathrm{D}}\right)}, \\
c_{\mathrm{R}-\mathrm{D}}^{(l n)} & =e^{j(2 \pi / \lambda)\left\{R_{\mathrm{D}} \cos \left(\phi_{\mathrm{D}}^{(n)}-\gamma_{\mathrm{D}}\right)-R_{\mathrm{R}} \cos \left(\phi_{\mathrm{R}-\mathrm{D}}^{(l)}-\gamma_{\mathrm{D}}\right)\right\}}, \\
\theta_{\mathrm{R}-\mathrm{D}} & =-\frac{2 \pi}{\lambda}\left(R_{\mathrm{R}}+D_{\mathrm{R}-\mathrm{D}}+R_{\mathrm{D}}\right), \\
f_{\mathrm{R}-\mathrm{D}}^{(l)} & =f_{\mathrm{R}_{\max }} \cos \left(\phi_{\mathrm{R}-\mathrm{D}}^{(l)}-\alpha_{\mathrm{R}}\right), \\
f_{\mathrm{D}}^{(n)} & =f_{\mathrm{D}_{\text {max }}} \cos \left(\phi_{\mathrm{D}}^{(n)}-\alpha_{\mathrm{D}}\right),
\end{aligned}
$$

where $f_{\mathrm{D}_{\max }}=v_{\mathrm{D}} / \lambda$ is the maximum Doppler frequency caused by the movement of the destination mobile station. The symbol $\gamma_{\mathrm{D}}$ in $(7 \mathrm{~d})$ refers to the position of the mobile relay with respect to the destination mobile station (see Figure 1). It is worth highlighting that the AOD $\phi_{\mathrm{R}-\mathrm{D}}^{(l)}$ and the $\mathrm{AOA} \phi_{\mathrm{D}}^{(n)}$ are independent random variables. Also keep in mind that $\phi_{\mathrm{R}-\mathrm{D}}^{(l)}$ and $\phi_{\mathrm{D}}^{(n)}$ are determined by the distribution of the local scatterers around the mobile relay and the destination mobile station, respectively.

One can show that the diffuse components $h_{\mathrm{R}-\mathrm{D}}^{(i q)}(t)(i, q=$ $1,2)$ of the remaining transmission links from the mobile relay antenna element $A_{\mathrm{R}}^{(q)}$ to the destination mobile station antenna element $A_{\mathrm{D}}^{(i)}$ can similarly be realized as described for the $A_{\mathrm{S}}^{(q)}-A_{\mathrm{R}}^{(i)}$ transmission link.

Finally, substituting (1) in (5) allows us to identify the overall fading channel between the source mobile station and the destination mobile station as

$$
\begin{aligned}
\mathbf{R}(t) & =\mathbf{H}_{\mathrm{R}-\mathrm{D}}(t) \mathbf{H}_{\mathrm{S}-\mathrm{R}}(t) \mathbf{S}(t)+\mathbf{H}_{\mathrm{R}-\mathrm{D}}(t) \mathbf{N}_{\mathrm{R}}(t)+\mathbf{N}_{\mathrm{D}}(t) \\
& =\mathbf{H}_{\mathrm{S}-\mathrm{R}-\mathrm{D}}(t) \mathbf{S}(t)+\mathbf{N}_{\mathrm{R}-\mathrm{D}}(t),
\end{aligned}
$$

where $\mathbf{N}_{\mathrm{R}-\mathrm{D}}(t)=\mathbf{H}_{\mathrm{R}-\mathrm{D}}(t) \mathbf{N}_{\mathrm{R}}(t)+\mathbf{N}_{\mathrm{D}}(t)$ is the total noise of the system. The symbol $\mathbf{H}_{\mathrm{S}-\mathrm{R}-\mathrm{D}}(t)$ denotes the overall channel matrix, which is defined as follows:

$$
\begin{aligned}
\mathbf{H}_{\mathrm{S}-\mathrm{R}-\mathrm{D}}(t) & =\mathbf{H}_{\mathrm{R}-\mathrm{D}}(t) \mathbf{H}_{\mathrm{S}-\mathrm{R}}(t) \\
& =\left(\begin{array}{ll}
h_{\mathrm{R}-\mathrm{D}}^{(11)}(t) & h_{\mathrm{R}-\mathrm{D}}^{(12)}(t) \\
h_{\mathrm{R}-\mathrm{D}}^{(21)}(t) & h_{\mathrm{R}-\mathrm{D}}^{(22)}(t)
\end{array}\right)\left(\begin{array}{ll}
h_{\mathrm{S}-\mathrm{R}}^{(11)}(t) & h_{\mathrm{S}-\mathrm{R}}^{(12)}(t) \\
h_{\mathrm{S}-\mathrm{R}}^{(21)}(t) & h_{\mathrm{S}-\mathrm{R}}^{(22)}(t)
\end{array}\right) \\
& =\left(\begin{array}{ll}
h_{\mathrm{S}-\mathrm{R}-\mathrm{D}}^{(11)}(t) & h_{\mathrm{S}-\mathrm{R}-\mathrm{D}}^{(12)}(t) \\
h_{\mathrm{S}-\mathrm{R}-\mathrm{D}}^{(21)}(t) & h_{\mathrm{S}-\mathrm{R}-\mathrm{D}}^{(22)}(t)
\end{array}\right) .
\end{aligned}
$$

It is noteworthy that the overall channel matrix $\mathbf{H}_{\mathrm{S}-\mathrm{R}-\mathrm{D}}(t)$ describes completely the reference model of the proposed geometrical three-ring MIMO M2M fading channel. Here, each element $h_{\mathrm{S}-\mathrm{R}-\mathrm{D}}^{(i q)}(t)(i, q=1,2)$ of the channel matrix defines the diffuse component of the overall MIMO M2M 
fading channel, describing the transmission link from the source mobile station antenna element $A_{\mathrm{S}}^{(q)}$ to the destination mobile station antenna element $A_{\mathrm{D}}^{(i)}$ via the mobile relay antenna elements. Expanding (9) allows us to explicitly write the diffuse component $h_{\mathrm{S}-\mathrm{R}-\mathrm{D}}^{(11)}(t)$ of the transmission link from the first antenna element at the source mobile station, $A_{\mathrm{S}}^{(1)}$, to the the first antenna element at the destination mobile station, $A_{\mathrm{D}}^{(1)}$, as follows:

$$
\begin{aligned}
& h_{\mathrm{S}-\mathrm{R}-\mathrm{D}}^{(11)}(t) \\
& =h_{\mathrm{R}-\mathrm{D}}^{(11)}(t) h_{\mathrm{S}-\mathrm{R}}^{(11)}(t)+h_{\mathrm{R}-\mathrm{D}}^{(12)}(t) h_{\mathrm{S}-\mathrm{R}}^{(21)}(t) \\
& =\lim _{\substack { K \rightarrow \infty \\
L \rightarrow \infty \\
\begin{subarray}{c}{M \rightarrow \infty \\
N \rightarrow \infty{ K \rightarrow \infty \\
L \rightarrow \infty \\
\begin{subarray} { c } { M \rightarrow \infty \\
N \rightarrow \infty } }\end{subarray}} \frac{2}{\sqrt{K L M N}} \sum_{k=1}^{K} \sum_{l=1}^{L} \sum_{m=1}^{M} \sum_{n=1}^{N} a_{\mathrm{S}}^{(m)} b_{\mathrm{D}}^{(n)} c_{\mathrm{S}-\mathrm{R}}^{(m k)} c_{\mathrm{R}-\mathrm{D}}^{(l n)} \Re\left\{b_{\mathrm{R}}^{(k)} a_{\mathrm{R}}^{(l)}\right\} \\
& \quad \times e^{j\left[2 \pi\left(f_{\mathrm{S}}^{(m)}+f_{\mathrm{S}-\mathrm{R}}^{(k)}+f_{\mathrm{R}-\mathrm{D}}^{(l)}+f_{\mathrm{D}}^{(n)}\right) t+\left(\theta_{\mathrm{S}-\mathrm{R}}^{(m k)}+\theta_{\mathrm{R}-\mathrm{D}}^{(l n)}+\theta_{\mathrm{S}-\mathrm{R}}+\theta_{\mathrm{R}-\mathrm{D}}\right)\right]},
\end{aligned}
$$

where $\Re\left\{b_{\mathrm{R}}^{(k)} a_{\mathrm{R}}^{(l)}\right\}$ denotes the real part of a complex number, that is, $2 \mathfrak{R}\left\{b_{\mathrm{R}}^{(k)} a_{\mathrm{R}}^{(l)}\right\}=b_{\mathrm{R}}^{(k)} a_{\mathrm{R}}^{(l)}+b_{\mathrm{R}}^{(k) *} a_{\mathrm{R}}^{(l) *}$. Note that the phases $\theta_{\mathrm{S}-\mathrm{R}}$ and $\theta_{\mathrm{R}-\mathrm{D}}$ in (10) are constant quantities, which can be set to zero without loss of generality since the statistical properties of the reference model are not influenced by a constant phase shift. Similarly, the diffuse component $h_{\mathrm{S}-\mathrm{R}-\mathrm{D}}^{(22)}(t)$ of the $A_{\mathrm{S}}^{(2)}-A_{\mathrm{D}}^{(2)}$ transmission link can be expressed as

$$
\begin{aligned}
& h_{\mathrm{S}-\mathrm{R}-\mathrm{D}}^{(22)}(t) \\
& =h_{\mathrm{R}-\mathrm{D}}^{(21)}(t) h_{\mathrm{S}-\mathrm{R}}^{(12)}(t)+h_{\mathrm{R}-\mathrm{D}}^{(22)}(t) h_{\mathrm{S}-\mathrm{R}}^{(22)}(t) \\
& =\lim _{\substack { K \rightarrow \infty \\
\begin{subarray}{c}{M \rightarrow \infty \\
N \rightarrow \infty{ K \rightarrow \infty \\
\begin{subarray} { c } { M \rightarrow \infty \\
N \rightarrow \infty } }\end{subarray}} \frac{2}{\sqrt{K L M N}} \sum_{k=1}^{K} \sum_{l=1}^{L} \sum_{m=1}^{M} \sum_{n=1}^{N} a_{\mathrm{S}}^{(m) *} b_{\mathrm{D}}^{(n) *} c_{\mathrm{S}-\mathrm{R}}^{(m k)} c_{\mathrm{R}-\mathrm{D}}^{(l n)} \mathfrak{R}\left\{b_{\mathrm{R}}^{(k)} a_{\mathrm{R}}^{(l)}\right\} \\
& \quad \times e^{-j\left[2 \pi\left(f_{\mathrm{S}}^{(m)}-f_{\mathrm{S}-\mathrm{R}}^{(k)}+f_{\mathrm{R}-\mathrm{D}}^{(l)}-f_{\mathrm{D}}^{(n)}\right) t-\left(\theta_{\mathrm{S}-\mathrm{R}}^{(m)}+\theta_{\mathrm{R}-\mathrm{D}}^{(l n)}+\theta_{\mathrm{S}-\mathrm{R}}+\theta_{\mathrm{R}-\mathrm{D})}\right)\right.} .
\end{aligned}
$$

The equations (10) and (11) will be used in the next subsection to calculate the $4 \mathrm{D}$ space-time CCF.

3.2. Correlation Properties of the Reference Model. By definition, the 4D space-time CCF between the transmission links $A_{\mathrm{S}}^{(1)}-A_{\mathrm{D}}^{(1)}$ and $A_{\mathrm{S}}^{(2)}-A_{\mathrm{D}}^{(2)}$ is equivalent to the correlation between the diffuse components $h_{\mathrm{S}-\mathrm{R}-\mathrm{D}}^{(11)}(t)$ and $h_{\mathrm{S}-\mathrm{R}-\mathrm{D}}^{(22)}(t)$, that is, [26]

$$
\rho_{11,22}\left(\delta_{\mathrm{S}}, \delta_{\mathrm{R}}, \delta_{\mathrm{D}}, \tau\right):=E\left\{h_{\mathrm{S}-\mathrm{R}-\mathrm{D}}^{(11)}(t) h_{\mathrm{S}-\mathrm{R}-\mathrm{D}}^{(22)^{*}}(t+\tau)\right\}
$$

It should be noticed here that the expectation operator is applied to all random variables, that is, the random phase shifts $\left\{\theta_{\mathrm{S}-\mathrm{R}}^{(m k)}, \theta_{\mathrm{R}-\mathrm{D}}^{(l n)}\right\}$, the AODs $\left\{\phi_{\mathrm{S}}^{(m)}, \phi_{\mathrm{R}-\mathrm{D}}^{(l)}\right\}$, and the AOAs
$\left\{\phi_{S-R}^{(k)}, \phi_{\mathrm{D}}^{(n)}\right\}$. Substituting (10) and (11) in (12), the 4D spacetime CCF can be expressed as

$$
\begin{aligned}
& \rho_{11,22}\left(\delta_{\mathrm{S}}, \delta_{\mathrm{R}}, \delta_{\mathrm{D}}, \tau\right) \\
& =\lim _{\substack{K \rightarrow \infty \\
M \rightarrow \infty \\
L \rightarrow \infty \\
N \rightarrow \infty}} \frac{4}{K L M N} \sum_{k=1}^{K} \sum_{l=1}^{L} \sum_{m=1}^{M} \sum_{n=1}^{N} E\left\{a_{\mathrm{S}}^{(m) 2} b_{\mathrm{D}}^{(n) 2}\left[\Re\left\{b_{\mathrm{R}}^{(k)} a_{\mathrm{R}}^{(l)}\right\}\right]^{2}\right. \\
& \left.\times e^{-j 2 \pi\left(f_{\mathrm{S}}^{(m)}+f_{\mathrm{S}-\mathrm{R}}^{(k)}+f_{\mathrm{R}-\mathrm{D}}^{(l)}+f_{\mathrm{D}}^{(n)}\right) \tau}\right\} .
\end{aligned}
$$

It is important to highlight, here, the functions of random variables to which the expectation is applied. We can see that $\left\{a_{\mathrm{S}}^{(m)}, f_{\mathrm{S}}^{(m)}\right\}$ and $\left\{a_{\mathrm{R}}^{(l)}, f_{\mathrm{R}-\mathrm{D}}^{(l)}\right\}$ are functions of the AODs $\phi_{\mathrm{S}}^{(m)}$ and $\phi_{\mathrm{R}-\mathrm{D}}^{(l)}$, respectively. While $\left\{b_{\mathrm{R}}^{(k)}, f_{\mathrm{S}-\mathrm{R}}^{(k)}\right\}$ and $\left\{b_{\mathrm{D}}^{(n)}, f_{\mathrm{D}}^{(n)}\right\}$ are functions of the AOAs $\phi_{S-R}^{(k)}$ and $\phi_{D}^{(n)}$, respectively [30]. If the number of local scatterers approaches infinity, that is, $K, L, M, N \rightarrow \infty$, then the discrete random variables $\phi_{\mathrm{S}}^{(m)}$, $\phi_{\mathrm{S}-\mathrm{R}}^{(k)}, \phi_{\mathrm{R}-\mathrm{D}}^{(l)}$, and $\phi_{\mathrm{D}}^{(n)}$ become continuous random variables $\phi_{S}, \phi_{S-R}, \phi_{R-D}$, and $\phi_{D}$, each of which is characterized by a certain distribution, denoted by $p_{\phi_{S}}\left(\phi_{S}\right), p_{\phi_{S-R}}\left(\phi_{S-R}\right)$, $p_{\phi_{\mathrm{R}-\mathrm{D}}}\left(\phi_{\mathrm{R}-\mathrm{D}}\right)$, and $p_{\phi_{\mathrm{D}}}\left(\phi_{\mathrm{D}}\right)$, respectively, [30]. The infinitesimal power of the diffuse components corresponding to the differential angles $d \phi_{\mathrm{S}}, d \phi_{\mathrm{S}-\mathrm{R}}, d \phi_{\mathrm{R}-\mathrm{D}}$, and $d \phi_{\mathrm{D}}$ is proportional to $p_{\phi_{\mathrm{S}}}\left(\phi_{\mathrm{S}}\right) p_{\phi_{\mathrm{S}-\mathrm{R}}}\left(\phi_{\mathrm{S}-\mathrm{R}}\right) p_{\phi_{\mathrm{R}-\mathrm{D}}}\left(\phi_{\mathrm{R}-\mathrm{D}}\right) p_{\phi_{\mathrm{D}}}\left(\phi_{\mathrm{D}}\right) d \phi_{\mathrm{S}} d \phi_{\mathrm{S}-\mathrm{R}} d \phi_{\mathrm{R}-\mathrm{D}} d \phi_{\mathrm{D}}$. This implies that when the number of local scatterers approaches infinity, that is, $K, L, M, N \rightarrow \infty$, the infinitesimal power of all diffuse components becomes equal to $1 /(K L M N)$, that is,

$$
\begin{aligned}
\frac{1}{K L M N}= & p_{\phi_{\mathrm{S}}}\left(\phi_{\mathrm{S}}\right) p_{\phi_{\mathrm{S}-\mathrm{R}}}\left(\phi_{\mathrm{S}-\mathrm{R}}\right) p_{\phi_{\mathrm{R}-\mathrm{D}}}\left(\phi_{\mathrm{R}-\mathrm{D}}\right) \\
& \times p_{\phi_{\mathrm{D}}}\left(\phi_{\mathrm{D}}\right) d \phi_{\mathrm{S}} d \phi_{\mathrm{S}-\mathrm{R}} d \phi_{\mathrm{R}-\mathrm{D}} d \phi_{\mathrm{D}}
\end{aligned}
$$

Thus, we can write the $4 \mathrm{D}$ space-time CCF $\rho_{11,22}\left(\delta_{\mathrm{S}}, \delta_{\mathrm{R}}\right.$, $\left.\delta_{\mathrm{D}}, \tau\right)$ of the reference model given in (13) as

$$
\rho_{11,22}\left(\delta_{\mathrm{S}}, \delta_{\mathrm{R}}, \delta_{\mathrm{D}}, \tau\right)=\rho_{\mathrm{S}}\left(\delta_{\mathrm{S}}, \tau\right) \cdot \rho_{\mathrm{R}}\left(\delta_{\mathrm{R}}, \tau\right) \cdot \rho_{\mathrm{D}}\left(\delta_{\mathrm{D}}, \tau\right),
$$

where

$$
\begin{aligned}
\rho_{\mathrm{S}}\left(\delta_{\mathrm{S}}, \tau\right)=\int_{-\pi}^{\pi} a_{\mathrm{S}}^{2}\left(\delta_{\mathrm{S}}, \phi_{\mathrm{S}}\right) e^{-j 2 \pi f_{\mathrm{S}}\left(\phi_{\mathrm{S}}\right) \tau} p_{\phi_{\mathrm{S}}}\left(\phi_{\mathrm{S}}\right) d \phi_{\mathrm{S}}, \\
\rho_{\mathrm{R}}\left(\delta_{\mathrm{R}}, \tau\right)=4 \iint_{-\pi}^{\pi} e^{-j 2 \pi\left[f_{\mathrm{S}-\mathrm{R}}\left(\phi_{S-\mathrm{R}}\right)+f_{\mathrm{R}-\mathrm{D}}\left(\phi_{\mathrm{R}-\mathrm{D}}\right)\right] \tau} \\
\quad \times\left[\Re\left\{b_{\mathrm{R}}\left(\delta_{\mathrm{R}}, \phi_{\mathrm{S}-\mathrm{R}}\right) a_{\mathrm{R}}\left(\delta_{\mathrm{R}}, \phi_{\mathrm{R}-\mathrm{D}}\right)\right\}\right]^{2} \\
\quad \times p_{\phi_{\mathrm{S}-\mathrm{R}}}\left(\phi_{\mathrm{S}-\mathrm{R}}\right) p_{\phi_{\mathrm{R}-\mathrm{D}}}\left(\phi_{\mathrm{R}-\mathrm{D}}\right) d \phi_{\mathrm{S}-\mathrm{R}} d \phi_{\mathrm{R}-\mathrm{D}}, \\
\rho_{\mathrm{D}}\left(\delta_{\mathrm{D}}, \tau\right)=\int_{-\pi}^{\pi} b_{\mathrm{D}}^{2}\left(\delta_{\mathrm{D}}, \phi_{\mathrm{D}}\right) e^{-j 2 \pi f_{\mathrm{D}}\left(\phi_{\mathrm{D}}\right) \tau} p_{\phi_{\mathrm{D}}}\left(\phi_{\mathrm{D}}\right) d \phi_{\mathrm{D}}
\end{aligned}
$$


are the CFs at the source mobile station, mobile relay, and the destination mobile station. Here,

$$
\begin{aligned}
a_{\mathrm{S}}\left(\delta_{\mathrm{S}}, \phi_{\mathrm{S}}\right) & =e^{j(\pi / \lambda) \delta_{\mathrm{S}} \cos \left(\phi_{\mathrm{S}}-\beta_{\mathrm{S}}\right)}, \\
b_{\mathrm{R}}\left(\delta_{\mathrm{R}}, \phi_{\mathrm{S}-\mathrm{R}}\right) & =e^{j(\pi / \lambda) \delta_{\mathrm{R}} \cos \left(\phi_{\mathrm{S}-\mathrm{R}}-\beta_{\mathrm{R}}\right)}, \\
a_{\mathrm{R}}\left(\delta_{\mathrm{R}}, \phi_{\mathrm{S}-\mathrm{R}}\right) & =e^{j(\pi / \lambda) \delta_{\mathrm{R}} \cos \left(\phi_{\mathrm{R}-\mathrm{D}}-\beta_{\mathrm{R}}\right)}, \\
b_{\mathrm{D}}\left(\delta_{\mathrm{D}}, \phi_{\mathrm{D}}\right) & =e^{j(\pi / \lambda) \delta_{\mathrm{D}} \cos \left(\phi_{\mathrm{D}}-\beta_{\mathrm{D}}\right)}, \\
f_{\mathrm{S}}\left(\phi_{\mathrm{S}}\right) & =f_{\mathrm{S}_{\max }} \cos \left(\phi_{\mathrm{S}}-\alpha_{\mathrm{S}}\right), \\
f_{\mathrm{S}-\mathrm{R}}\left(\phi_{\mathrm{S}-\mathrm{R}}\right) & =f_{\mathrm{R}_{\max }} \cos \left(\phi_{\mathrm{S}-\mathrm{R}}-\alpha_{\mathrm{R}}\right), \\
f_{\mathrm{R}-\mathrm{D}}\left(\phi_{\mathrm{R}-\mathrm{D}}\right) & =f_{\mathrm{R}_{\max }} \cos \left(\phi_{\mathrm{R}-\mathrm{D}}-\alpha_{\mathrm{R}}\right), \\
f_{\mathrm{D}}\left(\phi_{\mathrm{D}}\right) & =f_{\mathrm{D}_{\max }} \cos \left(\phi_{\mathrm{D}}-\alpha_{\mathrm{D}}\right) .
\end{aligned}
$$

In this article, we refer to the CF at the source mobile station $\rho_{\mathrm{S}}\left(\delta_{\mathrm{S}}, \tau\right)$ as the source CF. Likewise, the CF at the mobile relay (destination mobile station) $\rho_{\mathrm{S}}\left(\delta_{\mathrm{R}}, \tau\right)\left(\rho_{\mathrm{S}}\left(\delta_{\mathrm{D}}, \tau\right)\right)$ is termed as the relay $\mathrm{CF}$ (destination $\mathrm{CF}$ ). Equation (15) illustrates that the $4 \mathrm{D}$ space-time CCF $\rho_{11,22}\left(\delta_{\mathrm{S}}, \delta_{\mathrm{R}}, \delta_{\mathrm{D}}, \tau\right)$ of the reference model can be expressed as the product of the source CF $\rho_{\mathrm{S}}\left(\delta_{\mathrm{S}}, \tau\right)$, the relay CF $\rho_{\mathrm{R}}\left(\delta_{\mathrm{R}}, \tau\right)$, and the destination $\mathrm{CF}$ $\rho_{\mathrm{D}}\left(\delta_{\mathrm{D}}, \tau\right)$. Besides, from (16) and (18), it turns out that the source mobile station and the destination mobile station are interchangeable.

The $3 \mathrm{D}$ spatial CCF $\rho\left(\delta_{\mathrm{S}}, \delta_{\mathrm{R}}, \delta_{\mathrm{D}}\right)$, defined as $\rho\left(\delta_{\mathrm{S}}, \delta_{\mathrm{R}}\right.$, $\left.\delta_{\mathrm{D}}\right)=E\left\{h_{\mathrm{S}-\mathrm{R}-\mathrm{D}}^{(11)}(t) h_{\mathrm{S}-\mathrm{R}-\mathrm{D}}^{(22)^{*}}(t)\right\}$, is equal to the $4 \mathrm{D}$ space-time CCF $\rho_{11,22}\left(\delta_{\mathrm{S}}, \delta_{\mathrm{R}}, \delta_{\mathrm{D}}, \tau\right)$ at $\tau=0$, that is,

$$
\begin{aligned}
\rho\left(\delta_{\mathrm{S}}, \delta_{\mathrm{R}}, \delta_{\mathrm{D}}\right) & =\rho_{11,22}\left(\delta_{\mathrm{S}}, \delta_{\mathrm{R}}, \delta_{\mathrm{D}}, 0\right) \\
& =\rho_{\mathrm{S}}\left(\delta_{\mathrm{S}}, 0\right) \cdot \rho_{\mathrm{R}}\left(\delta_{\mathrm{R}}, 0\right) \cdot \rho_{\mathrm{D}}\left(\delta_{\mathrm{D}}, 0\right) .
\end{aligned}
$$

The temporal ACF $r_{h_{S-R-D}^{(i q)}}(\tau)$ of the diffuse component $h_{\mathrm{S}-\mathrm{R}-\mathrm{D}}^{(i q)}(t)$ of the transmission link from the source mobile station antenna element $A_{\mathrm{S}}^{(q)}$ to the destination mobile station antenna element $A_{\mathrm{D}}^{(i)}$, for all $i, q \in\{1,2\}$ can be given as

$$
r_{h_{\mathrm{S}-\mathrm{R}-\mathrm{D}}^{(i q)}}(\tau):=E\left\{h_{\mathrm{S}-\mathrm{R}-\mathrm{D}}^{(i q)}(t) h_{\mathrm{S}-\mathrm{R}-\mathrm{D}}^{(i q)^{*}}(t+\tau)\right\}
$$

It is not difficult to show that the temporal ACF $r_{h_{S-R-D}^{(i q)}}(\tau)$, for all $i, q \in\{1,2\}$ is equal to the $4 \mathrm{D}$ space-time CCF $\rho_{11,22}\left(\delta_{\mathrm{S}}, \delta_{\mathrm{R}}, \delta_{\mathrm{D}}, \tau\right)$ of the reference model at $\delta_{\mathrm{S}}=\delta_{\mathrm{R}}=\delta_{\mathrm{D}}=$ 0 , that is,

$$
\begin{aligned}
r_{h_{\mathrm{S}-\mathrm{R}-\mathrm{D}}^{(\mathrm{i})}}(\tau) & =\rho_{11,22}(0,0,0, \tau) \\
& =\rho_{\mathrm{S}}(0, \tau) \cdot \rho_{\mathrm{R}}(0, \tau) \cdot \rho_{\mathrm{D}}(0, \tau) .
\end{aligned}
$$

Substituting $\delta_{\mathrm{S}}=0, \delta_{\mathrm{R}}=0$, and $\delta_{\mathrm{D}}=0$ in (16), (17), and $(18)$, respectively, gives $\rho_{S}(0, \tau), \rho_{\mathrm{R}}(0, \tau)$, and $\rho_{\mathrm{D}}(0, \tau)$, respectively, as

$$
\begin{aligned}
& \rho_{\mathrm{S}}(0, \tau)=\int_{-\pi}^{\pi} e^{-j 2 \pi f_{\mathrm{S}}\left(\phi_{\mathrm{S}}\right) \tau} p_{\phi_{\mathrm{S}}}\left(\phi_{\mathrm{S}}\right) d \phi_{\mathrm{S}}, \\
& \rho_{\mathrm{R}}(0, \tau)=4 \iint_{-\pi}^{\pi} e^{-j 2 \pi\left[f_{\mathrm{S}-\mathrm{R}}\left(\phi_{\mathrm{S}-\mathrm{R}}\right)+f_{\mathrm{R}-\mathrm{D}}\left(\phi_{\mathrm{R}-\mathrm{D}}\right)\right] \tau} \\
& \times p_{\phi_{S-\mathrm{R}}}\left(\phi_{\mathrm{S}-\mathrm{R}}\right) p_{\phi_{\mathrm{R}-\mathrm{D}}}\left(\phi_{\mathrm{R}-\mathrm{D}}\right) d \phi_{\mathrm{S}-\mathrm{R}} d \phi_{\mathrm{R}-\mathrm{D}}, \\
& \rho_{\mathrm{D}}(0, \tau)=\int_{-\pi}^{\pi} e^{-j 2 \pi f_{\mathrm{D}}\left(\phi_{\mathrm{D}}\right) \tau} p_{\phi_{\mathrm{D}}}\left(\phi_{\mathrm{D}}\right) d \phi_{\mathrm{D}} .
\end{aligned}
$$

The reference model developed in Section 3 is a theoretical model, which is based on the assumption that the number of local scatterers $(K, L, M, N)$ around the source mobile station, the mobile relay, and the destination mobile station is infinite. The assumption of an infinite number of local scatterers prevents the realization of the reference model. However, a realizable stochastic simulation model can be derived from the reference model by: (i) using only a limited number of local scatterers $K=L, M$, and $N$ is finite, (ii) setting the constant phase shifts $\theta_{\mathrm{S}-\mathrm{R}}$ and $\theta_{\mathrm{R}-\mathrm{D}}$ to zero in (10), and (iii) considering the discrete AOD $\phi_{S}^{(m)}$ and $\phi_{\mathrm{R}-\mathrm{D}}^{(l)}$, as well as the AOA $\phi_{\mathrm{S}-\mathrm{R}}^{(k)}$ and $\phi_{\mathrm{D}}^{(n)}$ are constant quantities [30]. Thus, using (10), the diffuse component $\hat{h}_{\mathrm{S}-\mathrm{R}-\mathrm{D}}^{(11)}(t)$ (throughout this paper, the caret is used for the stochastic simulation model) of the $A_{\mathrm{S}}^{(1)}-A_{\mathrm{D}}^{(1)}$ transmission link of the stochastic simulation model can be expressed as

$$
\begin{aligned}
& \hat{h}_{\mathrm{S}-\mathrm{R}-\mathrm{D}}^{(11)}(t) \\
& =\frac{2}{\sqrt{K L M N}} \sum_{k=1}^{K} \sum_{l=1}^{L} \sum_{m=1}^{M} \sum_{n=1}^{N} a_{\mathrm{S}}^{(m)} b_{\mathrm{D}}^{(n)} c_{\mathrm{S}-\mathrm{R}}^{(m k)} c_{\mathrm{R}-\mathrm{D}}^{(l n)} \Re\left\{b_{\mathrm{R}}^{(k)} a_{\mathrm{R}}^{(l)}\right\} \\
& \quad \times e^{j\left[2 \pi\left\{f_{\mathrm{S}}^{(m)}+f_{S-\mathrm{R}}^{(k)}+f_{\mathrm{R}-\mathrm{D}}^{(l)}+f_{\mathrm{D}}^{(n)}\right\} t-\theta_{\mathrm{S}-\mathrm{R}}^{(m)}-\theta_{\mathrm{R}-\mathrm{D}}^{(l n)}\right]} .
\end{aligned}
$$

In the same way, the diffuse component $\hat{h}_{\mathrm{S}-\mathrm{R}-\mathrm{D}}^{(22)}(t)$ of the $A_{\mathrm{S}}^{(2)}-$ $A_{\mathrm{D}}^{(2)}$ transmission link in the stochastic simulation model can be expressed as

$$
\begin{array}{r}
\hat{h}_{\mathrm{S}-\mathrm{R}-\mathrm{D}}^{(22)}(t) \\
=\frac{2}{\sqrt{K L M N}} \sum_{k=1}^{K} \sum_{l=1}^{L} \sum_{m=1}^{M} \sum_{n=1}^{N} a_{\mathrm{S}}^{(m) *} b_{\mathrm{D}}^{(n) *} c_{\mathrm{S}-\mathrm{R}}^{(m k)} c_{\mathrm{R}-\mathrm{D}}^{(l n)} \mathfrak{R}\left\{b_{\mathrm{R}}^{(k)} a_{\mathrm{R}}^{(l)}\right\} \\
\times e^{j\left[2 \pi\left\{f_{\mathrm{S}}^{(m)}+f_{\mathrm{S}-\mathrm{R}}^{(k)}+f_{\mathrm{R}-\mathrm{D}}^{(l)}+f_{\mathrm{D}}^{(n)}\right\} t-\theta_{\mathrm{S}-\mathrm{R}}^{(m k)}-\theta_{\mathrm{R}-\mathrm{D}}^{(l n)}\right]} .
\end{array}
$$

The 4D space-time CCF of the diffuse components $\hat{h}_{\mathrm{S}-\mathrm{R}-\mathrm{D}}^{(11)}(t)$ and $\hat{h}_{\mathrm{S}-\mathrm{R}-\mathrm{D}}^{(22)}(t)$ of the stochastic simulation model is defined by

$$
\hat{\rho}_{11,22}\left(\delta_{\mathrm{S}}, \delta_{\mathrm{R}}, \delta_{\mathrm{D}}, \tau\right):=E\left\{\hat{h}_{\mathrm{S}-\mathrm{R}-\mathrm{D}}^{(11)}(t) \hat{h}_{\mathrm{S}-\mathrm{R}-\mathrm{D}}^{(22)^{*}}(t+\tau)\right\},
$$


where the expectation operator now only applies on the random phases $\theta_{S-\mathrm{R}}^{(m k)}$ and $\theta_{\mathrm{R}-\mathrm{D}}^{(l n)}$. The substitution of (26) and (27) in (28) results in the following closed-form expression:

$$
\begin{aligned}
& \hat{\rho}_{11,22}\left(\delta_{\mathrm{S}}, \delta_{\mathrm{R}}, \delta_{\mathrm{D}}, \tau\right) \\
& =\frac{4}{K L M N} \sum_{k=1}^{K} \sum_{l=1}^{L} \sum_{m=1}^{M} \sum_{n=1}^{N} a_{\mathrm{S}}^{(m) 2} b_{\mathrm{D}}^{(n) 2}\left(\Re\left\{b_{\mathrm{R}}^{(k)} a_{\mathrm{R}}^{(l)}\right\}\right)^{2} \\
& \times e^{-j 2 \pi\left\{f_{\mathrm{S}}^{(m)}+f_{\mathrm{S}-\mathrm{R}}^{(k)}+f_{\mathrm{R}-\mathrm{D}}^{(l)}+f_{\mathrm{D}}^{(n)}\right\} \tau} \\
& =\hat{\rho}_{\mathrm{S}}\left(\delta_{\mathrm{S}}, \tau\right) \cdot \hat{\rho}_{\mathrm{R}}\left(\delta_{\mathrm{R}}, \tau\right) \cdot \hat{\rho}_{\mathrm{D}}\left(\delta_{\mathrm{D}}, \tau\right),
\end{aligned}
$$

where

$$
\begin{aligned}
& \hat{\rho}_{\mathrm{S}}\left(\delta_{\mathrm{S}}, \tau\right)=\frac{1}{M} \sum_{m=1}^{M} a_{\mathrm{S}}^{(m) 2}\left(\delta_{\mathrm{S}}\right) e^{-j 2 \pi f_{\mathrm{S}}^{(m)} \tau} \\
& \hat{\rho}_{\mathrm{R}}\left(\delta_{\mathrm{R}}, \tau\right)=\frac{4}{K L} \sum_{k=1}^{K} \sum_{l=1}^{L}\left(\Re\left\{b_{\mathrm{R}}^{(k)} a_{\mathrm{R}}^{(l)}\right\}\right)^{2} e^{-j 2 \pi\left[f_{\mathrm{S} \cdot \mathrm{R}}^{(k)}+f_{\mathrm{R}-\mathrm{D}}^{(l)}\right] \tau}, \\
& \hat{\rho}_{\mathrm{D}}\left(\delta_{\mathrm{D}}, \tau\right)=\frac{1}{N} \sum_{n=1}^{N} b_{\mathrm{D}}^{(n) 2}\left(\delta_{\mathrm{D}}\right) e^{-j 2 \pi f_{\mathrm{D}}^{(n)} \tau}
\end{aligned}
$$

are called the source $\mathrm{CF}$, the relay $\mathrm{CF}$, and the destination $\mathrm{CF}$ of the simulation model.

The $3 \mathrm{D}$ spatial CCF $\hat{\rho}\left(\delta_{\mathrm{S}}, \delta_{\mathrm{R}}, \delta_{\mathrm{D}}\right)$ of the simulation model, defined as

$$
\hat{\rho}\left(\delta_{\mathrm{S}}, \delta_{\mathrm{R}}, \delta_{\mathrm{D}}\right)=E\left\{\hat{h}_{\mathrm{S}-\mathrm{R}-\mathrm{D}}^{(11)}(t) \hat{h}_{\mathrm{S}-\mathrm{R}-\mathrm{D}}^{(222)}(t)\right\}
$$

is equal to the $4 \mathrm{D}$ space-time CCF $\hat{\rho}_{11,22}\left(\delta_{\mathrm{S}}, \delta_{\mathrm{R}}, \delta_{\mathrm{D}}, \tau\right)$ at $\tau=0$, that is,

$$
\begin{aligned}
\hat{\rho}\left(\delta_{\mathrm{S}}, \delta_{\mathrm{R}}, \delta_{\mathrm{D}}\right) & =\hat{\rho}_{11,22}\left(\delta_{\mathrm{S}}, \delta_{\mathrm{R}}, \delta_{\mathrm{D}}, 0\right) \\
& =\hat{\rho}_{\mathrm{S}}\left(\delta_{\mathrm{S}}, 0\right) \cdot \hat{\rho}_{\mathrm{R}}\left(\delta_{\mathrm{R}}, 0\right) \cdot \hat{\rho}_{\mathrm{D}}\left(\delta_{\mathrm{D}}, 0\right) .
\end{aligned}
$$

In the stochastic simulation model, the temporal ACF $\hat{r}_{h_{S-R-\mathrm{D}}^{(i q)}}(\tau)$ of the diffuse component $\hat{h}_{\mathrm{S}-\mathrm{R}-\mathrm{D}}^{(i q)}(t)$ of the $A_{\mathrm{S}}^{(q)}-A_{\mathrm{D}}^{(i)}$ transmission link can be derived as follows:

$$
\begin{aligned}
\hat{r}_{h_{S-\mathrm{R}-\mathrm{D}}^{(i q)}}(\tau) & :=E\left\{\hat{h}_{\mathrm{S}-\mathrm{R}-\mathrm{D}}^{(i q)}(t) \hat{h}_{\mathrm{S}-\mathrm{R}-\mathrm{D}}^{(i q)^{*}}(t+\tau)\right\} \\
& =\frac{1}{K L M N} \sum_{k=1}^{K} \sum_{l=1}^{L} \sum_{m=1}^{M} \sum_{n=1}^{N} e^{-j 2 \pi\left\{f_{\mathrm{S}}^{(m)}+f_{S-\mathrm{R}}^{(k)}+f_{\mathrm{R}-\mathrm{D}}^{(())}+f_{\mathrm{D}}^{(n)}\right\} \tau} \\
& =\hat{\rho}_{\mathrm{S}}(0, \tau) \cdot \hat{\rho}_{\mathrm{R}}(0, \tau) \cdot \hat{\rho}_{\mathrm{D}}(0, \tau) \quad \forall i, q \in\{1,2\} .
\end{aligned}
$$

From (35), it can be seen that the temporal ACF $\hat{r}_{h_{S-R-\mathrm{D}}(i)}(\tau)$ is equal to the $4 \mathrm{D}$ space-time CCF $\hat{\rho}_{11,22}\left(\delta_{S}, \delta_{\mathrm{R}}, \delta_{\mathrm{D}}, \tau\right)$ of the simulation model at $\delta_{\mathrm{S}}=\delta_{\mathrm{R}}=\delta_{\mathrm{D}}=0$, that is, $\hat{r}_{h_{S-\mathrm{R}-\mathrm{D}}}^{(i q)}(\tau)=$ $\hat{\rho}_{11,22}(0,0,0, \tau)$.

\section{Scattering Scenarios}

This section studies the correlation properties of the reference model under nonisotropic scattering conditions. In order to characterize nonisotropic scattering around the source mobile station (destination mobile station), we have utilized the von Mises distribution for the AOD $\phi_{S}\left(\mathrm{AOA} \phi_{\mathrm{D}}\right)$, that is,

$$
p_{\phi_{S}}\left(\phi_{S}\right)=\frac{1}{2 \pi I_{0}\left(\kappa_{S}\right)} e^{\kappa_{S} \cos \left(\phi_{S}-\phi_{S}^{(0)}\right)}, \quad \phi_{S} \in[0,2 \pi),
$$

where $I_{0}(\cdot)$ is the modified Bessel function of the first kind of order zero, the parameter $\phi_{\mathrm{S}}^{(0)}\left(\phi_{\mathrm{D}}^{(0)}\right) \in[0,2 \pi)$ is the mean AOD (AOA), and the parameter $\kappa_{\mathrm{S}}\left(\kappa_{\mathrm{D}}\right) \geq 0$ controls the angular spread of $\phi_{S}\left(\phi_{\mathrm{D}}\right)$. Similarly, nonisotropic scattering around the mobile relay can be defined by the von Mises distribution of the AOA $\phi_{S-\mathrm{R}}$ along with the von Mises distribution of the AOD $\phi_{\mathrm{R}-\mathrm{D}}$ over $[0,2 \pi)$. Hence, the distributions $p_{\phi_{S-R}}\left(\phi_{S-R}\right)$ and $p_{\phi_{R-D}}\left(\phi_{R-D}\right)$ can be obtained by replacing the index S by S-R and R-D in (36), respectively. The reason for using the von Mises distribution is its flexibility to closely approximate the Gaussian distribution and the cardioid distribution as well as to include the uniform distribution as a special case [35]. Moreover, Abdi et al. [36] made a proposition of employing the von Mises distribution to model AOA statistics of mobile radio fading channels. They supported their proposal by matching the von Mises distribution to the measured data. Substituting (36) in (16) and using [37, Equations (3.3384)] results in the following closed-form expression for the source CF:

$$
\begin{aligned}
& \rho_{\mathrm{S}}\left(\delta_{\mathrm{S}}, \tau\right) \\
& =\frac{1}{I_{0}\left(\kappa_{\mathrm{S}}\right)} I_{0} \\
& \quad \times\left(\left[\kappa_{\mathrm{S}}^{2}-4 \pi^{2}\left\{\left(\frac{\delta_{\mathrm{S}}}{\lambda}\right)^{2}+\left(f_{\mathrm{S}_{\max }} \tau\right)^{2}-2 \frac{\delta_{\mathrm{S}}}{\lambda} f_{\mathrm{S}_{\max }} \tau \cos \left(\alpha_{\mathrm{S}}-\beta_{\mathrm{S}}\right)\right\}\right.\right. \\
& \left.\left.\quad+j 4 \pi \kappa_{\mathrm{S}}\left\{\frac{\delta_{\mathrm{S}}}{\lambda} \cos \left(\beta_{\mathrm{S}}-\phi_{\mathrm{S}}^{(0)}\right)-f_{\mathrm{S}_{\max }} \tau \cos \left(\alpha_{\mathrm{S}}-\phi_{\mathrm{S}}^{(0)}\right)\right\}\right]^{1 / 2}\right) .
\end{aligned}
$$

The destination $\mathrm{CF} \rho_{\mathrm{D}}\left(\delta_{\mathrm{D}}, \tau\right)$ can easily be realized by replacing the index $\mathrm{S}$ by $\mathrm{D}$ in (37).

Likewise, substituting the von Mises distribution for the AOA $\phi_{S-R}$ and the AOD $\phi_{R-D}$ in (17) and solving the integrals using [37, Equations (3.338-4)], provides us with the closed-form solution for the relay $\mathrm{CF} \rho_{\mathrm{R}}\left(\delta_{\mathrm{R}}, \tau\right)$ as given in (38).

Substituting (37), (38), and the closed-form expression of $\rho_{\mathrm{D}}\left(\delta_{\mathrm{D}}, \tau\right)$ in $(15)$ results in the $4 \mathrm{D}$ space-time CCF $\rho_{11,22}\left(\delta_{\mathrm{S}}, \delta_{\mathrm{R}}, \delta_{\mathrm{D}}, \tau\right)$ of the reference model in a closed form. 


$$
\begin{aligned}
& \rho_{\mathrm{R}}\left(\delta_{\mathrm{R}}, \tau\right)=\left(\frac{1}{\left(I_{0}\left(\kappa_{\mathrm{S}-\mathrm{R}}\right) I_{0}\left(\kappa_{\mathrm{R}-\mathrm{D}}\right)\right)}\right) \\
& \times\left[I _ { 0 } \left(\left[\kappa_{\mathrm{S}-\mathrm{R}}^{2}-4 \pi^{2}\left\{\left(\frac{\delta_{\mathrm{R}}}{\lambda}\right)^{2}+\left(f_{\mathrm{R}_{\max }} \tau\right)^{2}-2 \frac{\delta_{\mathrm{R}}}{\lambda} f_{\mathrm{R}_{\max }} \tau \cos \left(\alpha_{\mathrm{R}}-\beta_{\mathrm{R}}\right)\right\}\right.\right.\right. \\
& \left.\left.+j 4 \pi \kappa_{\mathrm{S}-\mathrm{R}}\left\{\frac{\delta_{\mathrm{R}}}{\lambda} \cos \left(\beta_{\mathrm{R}}-\phi_{\mathrm{S}-\mathrm{R}}^{(0)}\right)-f_{\mathrm{R}_{\max }} \tau \cos \left(\alpha_{\mathrm{R}}-\phi_{\mathrm{S}-\mathrm{R}}^{(0)}\right)\right\}\right]^{1 / 2}\right) \\
& \times I_{0}\left(\left[\kappa_{\mathrm{R}-\mathrm{D}}^{2}-4 \pi^{2}\left\{\left(\frac{\delta_{\mathrm{R}}}{\lambda}\right)^{2}+\left(f_{\mathrm{R}_{\max }} \tau\right)^{2}-2 \frac{\delta_{\mathrm{R}}}{\lambda} f_{\mathrm{R}_{\max }} \tau \cos \left(\alpha_{\mathrm{R}}-\beta_{\mathrm{R}}\right)\right\}\right.\right. \\
& \left.\left.+j 4 \pi \kappa_{\mathrm{R}-\mathrm{D}}\left\{\frac{\delta_{\mathrm{R}}}{\lambda} \cos \left(\beta_{\mathrm{R}}-\phi_{\mathrm{R}-\mathrm{D}}^{(0)}\right)-f_{\mathrm{R}_{\max }} \tau \cos \left(\alpha_{\mathrm{R}}-\phi_{\mathrm{R}-\mathrm{D}}^{(0)}\right)\right\}\right]^{1 / 2}\right) \\
& +I_{0}\left(\left[\kappa_{\mathrm{S}-\mathrm{R}}^{2}-4 \pi^{2} f_{\mathrm{R}_{\max }}^{2} \tau^{2}-j 4 \pi \kappa_{\mathrm{S}-\mathrm{R}} f_{\mathrm{R}_{\max }} \tau \cos \left(\alpha_{\mathrm{R}}-\phi_{\mathrm{S}-\mathrm{R}}^{(0)}\right)\right]^{1 / 2}\right) \\
& \times I_{0}\left(\left[\kappa_{\mathrm{R}-\mathrm{D}}^{2}-4 \pi^{2} f_{\mathrm{R}_{\max }}^{2} \tau^{2}-j 4 \pi \kappa_{\mathrm{R}-\mathrm{D}} f_{\mathrm{R}_{\max }} \tau \cos \left(\alpha_{\mathrm{R}}-\phi_{\mathrm{R}-\mathrm{D}}^{(0)}\right)\right]^{1 / 2}\right) \\
& +I_{0}\left(\left[\kappa_{\mathrm{S}-\mathrm{R}}^{2}-4 \pi^{2}\left\{\left(\frac{\delta_{\mathrm{R}}}{\lambda}\right)^{2}+\left(f_{\mathrm{R}_{\max }} \tau\right)^{2}+2 \frac{\delta_{\mathrm{R}}}{\lambda} f_{\mathrm{R}_{\max }} \tau \cos \left(\alpha_{\mathrm{R}}-\beta_{\mathrm{R}}\right)\right\}\right.\right. \\
& \left.\left.-j 4 \pi \kappa_{\mathrm{S}-\mathrm{R}}\left\{\frac{\delta_{\mathrm{R}}}{\lambda} \cos \left(\beta_{\mathrm{R}}-\phi_{\mathrm{S}-\mathrm{R}}^{(0)}\right)+f_{\mathrm{R}_{\max }} \tau \cos \left(\alpha_{\mathrm{R}}-\phi_{\mathrm{S}-\mathrm{R}}^{(0)}\right)\right\}\right]^{1 / 2}\right) \\
& \times I_{o}\left(\left[\kappa_{\mathrm{R}-\mathrm{D}}^{2}-4 \pi^{2}\left\{\left(\frac{\delta_{\mathrm{R}}}{\lambda}\right)^{2}+\left(f_{\mathrm{R}_{\max }} \tau\right)^{2}+2 \frac{\delta_{\mathrm{R}}}{\lambda} f_{\mathrm{R}_{\max }} \tau \cos \left(\alpha_{\mathrm{R}}-\beta_{\mathrm{R}}\right)\right\}\right.\right. \\
& \left.\left.\left.-j 4 \pi \kappa_{\mathrm{R}-\mathrm{D}}\left\{\frac{\delta_{\mathrm{R}}}{\lambda} \cos \left(\beta_{\mathrm{R}}-\phi_{\mathrm{R}-\mathrm{D}}^{(0)}\right)+f_{\mathrm{R}_{\max }} \tau \cos \left(\alpha_{\mathrm{R}}-\phi_{\mathrm{R}-\mathrm{D}}^{(0)}\right)\right\}\right]^{1 / 2}\right)\right] .
\end{aligned}
$$

Note that the von Mises distribution reduces to the uniform distribution for $\kappa_{\mathrm{S}}=\kappa_{\mathrm{S}-\mathrm{R}}=\kappa_{\mathrm{R}-\mathrm{D}}=\kappa_{\mathrm{D}}=$ 0 . This implies that setting the respective $\kappa$ 's to zero reduces (37) and (38) to the CFs (i.e., [38, equation (27)] and [38, Equation (28)], respectively), derived for isotropic scattering conditions. From the $4 \mathrm{D}$ space-time CCF $\rho_{11,22}\left(\delta_{\mathrm{S}}, \delta_{\mathrm{R}}, \delta_{\mathrm{D}}, \tau\right)$ of the reference model derived for isotropic scattering conditions, it follows that the $3 \mathrm{D}$ spatial CCF $\rho\left(\delta_{\mathrm{S}}, \delta_{\mathrm{R}}, \delta_{\mathrm{D}}\right)$ of the reference model is a product of four Bessel functions, that is, $\rho\left(\delta_{\mathrm{S}}, \delta_{\mathrm{R}}, \delta_{\mathrm{D}}\right)=$ $\rho_{11,22}\left(\delta_{\mathrm{S}}, \delta_{\mathrm{R}}, \delta_{\mathrm{D}}, 0\right)=J_{0}\left(2 \pi \delta_{\mathrm{S}} / \lambda\right)\left\{2 J_{0}\left(2 \pi \delta_{\mathrm{R}} / \lambda\right)\right\}^{2} J_{0}\left(2 \pi \delta_{\mathrm{D}} / \lambda\right)$. In the same way, the temporal ACF $r_{h_{S-\mathrm{R}-\mathrm{D}}^{(i q)}}(\tau)$ of the reference model can be written as $r_{h_{\mathrm{S}-\mathrm{R}-\mathrm{D}}^{(i q)}}(\tau)=\rho_{11,22}(0,0,0, \tau)=$ $J_{0}\left(2 \pi f_{\mathrm{S}_{\max }} \tau\right)\left\{2 J_{0}\left(2 \pi f_{\mathrm{R}_{\max }} \tau\right)\right\}^{2} J_{0}\left(2 \pi f_{\mathrm{D}_{\max }} \tau\right)$. A product of two Bessel functions describes the $2 \mathrm{D}$ spatial CCF and the temporal ACF of the reference model derived from a geometrical two-ring scattering model [30]. For the 3D spatial CCF and the temporal ACF of the reference model derived from a geometrical three-ring scattering model, a product of four Bessel functions is justified, since the geometrical three-ring scattering model is a concatenation of two separate geometrical two-ring scattering models.

\section{Numerical Results}

The purpose of this section is to illustrate the important theoretical results found for the CCFs of the reference model and the stochastic simulation model by evaluating the expressions in (16), (18), (30), and (32). Here, we focus on discussing numerical results for the source CFs and the relay CFs. The results for the destination CFs can easily be obtained from the source CFs just by replacing the index $S$ by $\mathrm{D}$. As a performance criterion, we consider the absolute error $e_{S}\left(\delta_{S}, \tau\right)=\left|\rho_{S}\left(\delta_{S}, \tau\right)-\hat{\rho}_{S}\left(\delta_{S}, \tau\right)\right|$ as a measure for the quality of the approximation $\rho_{\mathrm{S}}\left(\delta_{\mathrm{S}}, \tau\right) \approx \hat{\rho}_{\mathrm{S}}\left(\delta_{\mathrm{S}}, \tau\right)$. Similarly, the absolute error $e_{\mathrm{R}}\left(\delta_{\mathrm{R}}, \tau\right)=\left|\rho_{\mathrm{R}}\left(\delta_{\mathrm{R}}, \tau\right)-\hat{\rho}_{\mathrm{R}}\left(\delta_{\mathrm{R}}, \tau\right)\right|$ has been introduced to study the amount of precision of the approximation $\rho_{\mathrm{R}}\left(\delta_{\mathrm{R}}, \tau\right) \approx \hat{\rho}_{\mathrm{R}}\left(\delta_{\mathrm{R}}, \tau\right)$. The selected values for the parameters influencing the CFs are: $\beta_{S}=\beta_{R}=\pi / 2$, $\alpha_{\mathrm{S}}=\pi / 4, \alpha_{\mathrm{R}}=0$, and $f_{\mathrm{S}_{\max }}=f_{\mathrm{R}_{\max }}=91 \mathrm{~Hz}$. The wavelength $\lambda$ was set to $\lambda=0.15 \mathrm{~m}$.

\section{The Stochastic Simulation Model}

For the stochastic simulation model, an appropriate number of discrete scatterers $M$ and $K(L)$ located on the rings around the source mobile station and the mobile relay, 


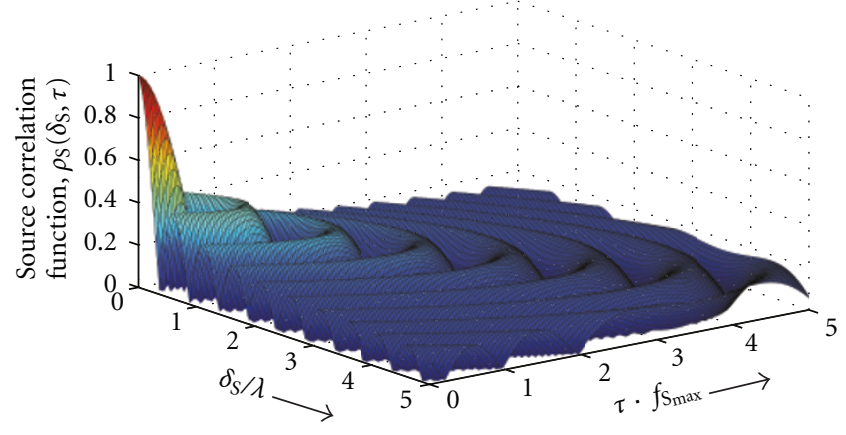

Figure 3: The source CF $\rho_{\mathrm{S}}\left(\delta_{\mathrm{S}}, \tau\right)$ of the $2 \times 2 \times 2$ MIMO M2M reference channel model under isotropic scattering conditions.

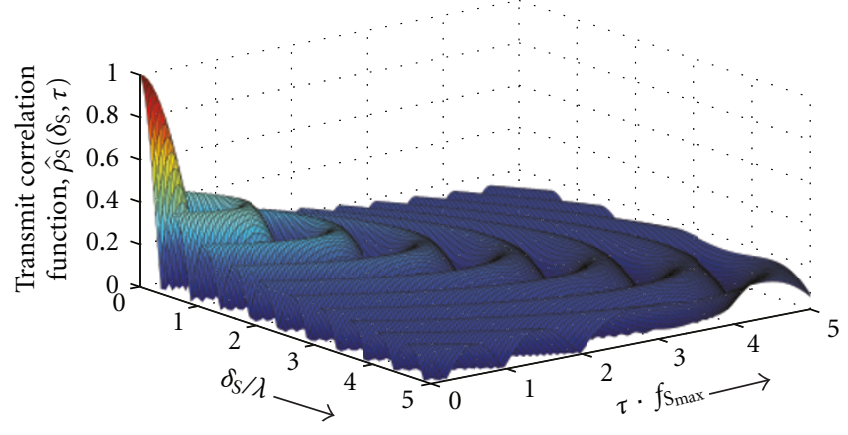

Figure 4: The source CF $\hat{\rho}_{\mathrm{S}}\left(\delta_{\mathrm{S}}, \tau\right)$ of the $2 \times 2 \times 2$ MIMO M2M stochastic channel simulator under isotropic scattering conditions.

respectively, should be selected. In our simulations, we have chosen $M=40$ and $K=L=23$. A good solution to the parameter computation problem in $\mathrm{M} 2 \mathrm{M}$ fading channel simulators in case of isotropic scattering is advanced in [30], where the authors have suggested the extended method of exact Doppler spread (EMEDS). Whereas, in case of nonisotropic scattering, a high-performance parameter computation method is the modified method of equal areas (MMEA) [39]. Since the MMEA reduces to the EMEDS in case of isotropic scattering [30], we have used the MMEA for computing the AODs $\phi_{\mathrm{S}}^{(m)}$ and $\phi_{\mathrm{R}-\mathrm{D}}^{(l)}$ as well as the AOA $\phi_{\mathrm{S}-\mathrm{R}}^{(k)}$.

Figures 3-8 bring to light the numerical results associated with isotropic scattering conditions. Figure 3 demonstrates the shape of the source $\mathrm{CF} \rho_{\mathrm{S}}\left(\delta_{\mathrm{S}}, \tau\right)$ of the reference model determined by (37) when $\kappa_{\mathrm{S}}=0$, whereas the simulation model's source CF $\hat{\rho}_{\mathrm{S}}\left(\delta_{\mathrm{S}}, \tau\right)$ is displayed in Figure 4 . The absolute error $e_{S}\left(\delta_{S}, \tau\right)$, presented in Figure 5, shows the quality of the approximation $\rho_{\mathrm{S}}\left(\delta_{\mathrm{S}}, \tau\right) \approx \hat{\rho}_{\mathrm{S}}\left(\delta_{\mathrm{S}}, \tau\right)$. The shape of the relay CF $\rho_{\mathrm{R}}\left(\delta_{\mathrm{R}}, \tau\right)$ of the reference model given by (38) when $\kappa_{\mathrm{R}}=0$ and the simulation model's relay CF $\hat{\rho}_{\mathrm{R}}\left(\delta_{\mathrm{R}}, \tau\right)$ are exhibited in Figures 6 and 7, respectively. A careful study of the absolute error $e_{\mathrm{R}}\left(\delta_{\mathrm{R}}, \tau\right)$ in Figure 8 reveals the ranges of $\delta_{\mathrm{R}} / \lambda$ and $\tau \cdot f_{\mathrm{R}_{\max }}$ with an excellent approximation $\rho_{\mathrm{R}}\left(\delta_{\mathrm{R}}, \tau\right) \approx \hat{\rho}_{\mathrm{R}}\left(\delta_{\mathrm{R}}, \tau\right)$. When $\delta_{\mathrm{R}} / \lambda$ is confined in the range $[0,(K=L) / 8]$, then the approximation $\rho_{\mathrm{R}}\left(\delta_{\mathrm{R}}, \tau\right) \approx \hat{\rho}_{\mathrm{R}}\left(\delta_{\mathrm{R}}, \tau\right)$ is very accurate. On the other hand, for $\delta_{\mathrm{R}}=0$ with $\tau \cdot f_{\mathrm{R}_{\max }}$

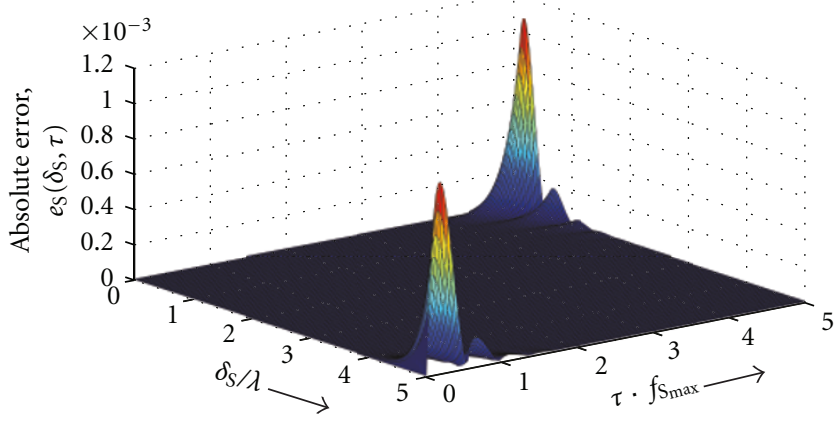

Figure 5: Absolute error $e_{S}\left(\delta_{\mathrm{S}}, \tau\right)=\left|\rho_{\mathrm{S}}\left(\delta_{\mathrm{S}}, \tau\right)-\hat{\rho}_{\mathrm{S}}\left(\delta_{\mathrm{S}}, \tau\right)\right|$ by using the MMEA with $M=40$ (isotropic scattering).

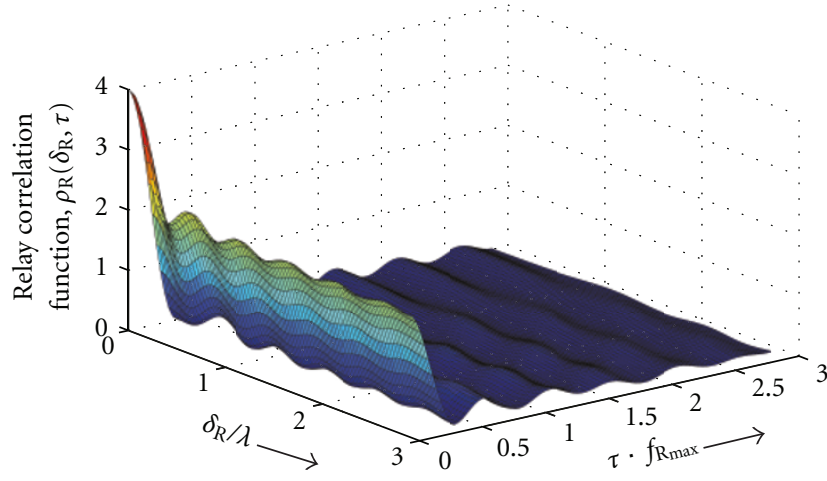

Figure 6: The relay CF $\rho_{\mathrm{R}}\left(\delta_{\mathrm{R}}, \tau\right)$ of the $2 \times 2 \times 2$ MIMO M2M reference channel model under isotropic scattering conditions.

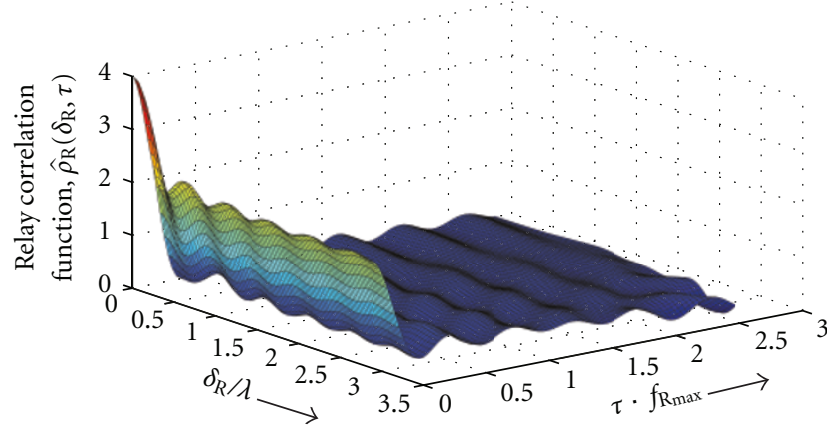

Figure 7: The relay CF $\hat{\rho}_{\mathrm{R}}\left(\delta_{\mathrm{R}}, \tau\right)$ of the $2 \times 2 \times 2$ MIMO M2M stochastic channel simulator under isotropic scattering conditions.

in the range $[0,(K=L) / 8]$, the absolute error $e_{\mathrm{R}}\left(\delta_{\mathrm{R}}, \tau\right)$ is almost zero.

Figures 9-14 elucidate the results in case of nonisotropic scattering. As mentioned in Section 4, the von Mises distribution has been employed to characterize nonisotropic scattering around the source mobile station (destination mobile station) and the mobile relay. In our simulations, the parameters of the von Mises distribution were set as $\phi_{\mathrm{S}}^{(0)}=\phi_{\mathrm{S}-\mathrm{R}}^{(0)}=\phi_{\mathrm{R}-\mathrm{D}}^{(0)}=60^{\circ}$ and $\kappa_{\mathrm{S}}=\kappa_{\mathrm{S}-\mathrm{R}}=\kappa_{\mathrm{R}-\mathrm{D}}=40$. Figure 9 has been included here to get a clear picture of the absolute value of the reference model's source CF $\left|\rho_{\mathrm{S}}\left(\delta_{\mathrm{S}}, \tau\right)\right|$ 


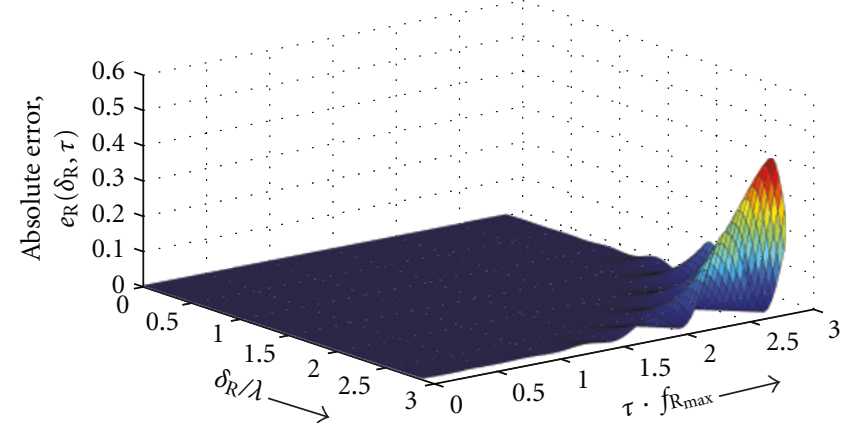

FIGURE 8: Absolute error $e_{\mathrm{R}}\left(\delta_{\mathrm{R}}, \tau\right)=\left|\rho_{\mathrm{R}}\left(\delta_{\mathrm{R}}, \tau\right)-\hat{\rho}_{\mathrm{R}}\left(\delta_{\mathrm{R}}, \tau\right)\right|$ by using the MMEA with $K=L=23$ (isotropic scattering).

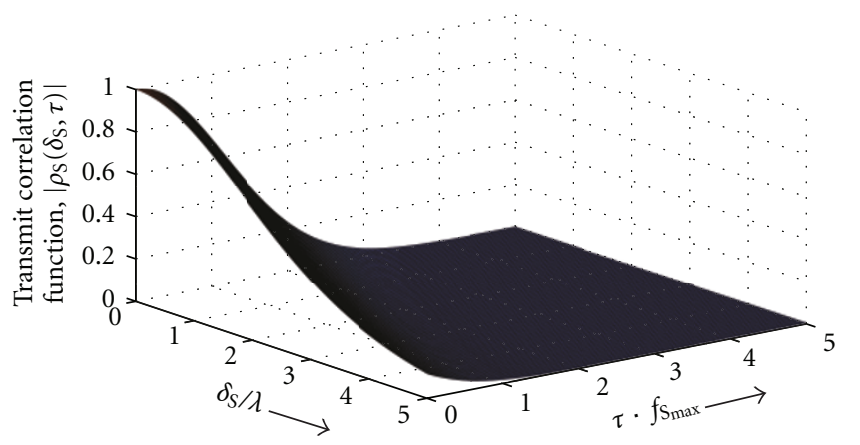

FIgURE 9: Absolute value of the source $\mathrm{CF}\left|\rho_{\mathrm{S}}\left(\delta_{\mathrm{S}}, \tau\right)\right|$ of the $2 \times$ $2 \times 2$ MIMO M2M reference channel model under nonisotropic scattering conditions (von Mises density with $\phi_{\mathrm{S}}^{(0)}=60^{\circ}$ and $\kappa_{\mathrm{S}}=$ 40).

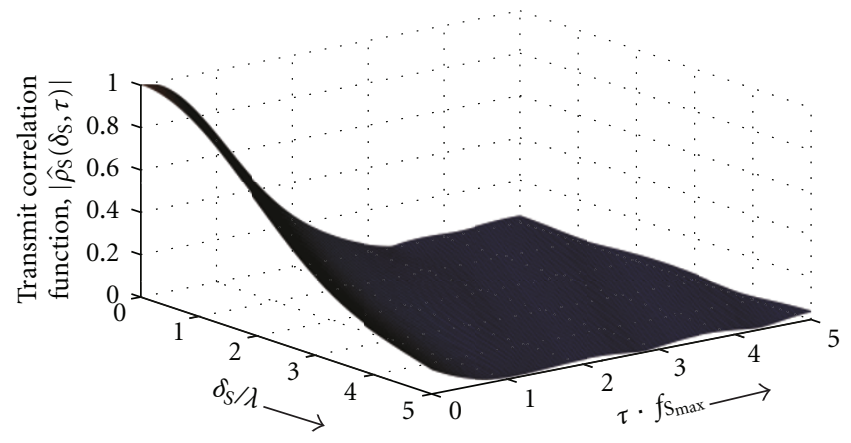

FIGURE 10: Absolute value of the source CF $\left|\hat{\rho}_{S}\left(\delta_{S}, \tau\right)\right|$ of the $2 \times 2 \times 2$ MIMO M2M stochastic channel simulator designed by applying the MMEA with $M=40$ (nonisotropic scattering, von Mises density with $\phi_{\mathrm{S}}^{(0)}=60^{\circ}$ and $\left.\kappa_{\mathrm{S}}=40\right)$.

given in (37), whereas Figure 10 provides information about the absolute value of the simulation model's source CF $\left|\hat{\rho}_{\mathrm{S}}\left(\delta_{\mathrm{S}}, \tau\right)\right|$. The absolute error $e_{\mathrm{S}}\left(\delta_{\mathrm{S}}, \tau\right)$ in Figure 11 shows that the approximation $\rho_{\mathrm{S}}\left(\delta_{\mathrm{S}}, \tau\right) \approx \hat{\rho}_{\mathrm{S}}\left(\delta_{\mathrm{S}}, \tau\right)$ holds for nonisotropic scattering as well. The error function $e_{\mathrm{S}}\left(\delta_{\mathrm{S}}, \tau\right)$ shows a ripple effect, where the maximum value of this error function is in the orders of $3 \cdot 10^{-2}$. It has been recommended in the literature to utilize the Lp-norm method (LPNM) [40]

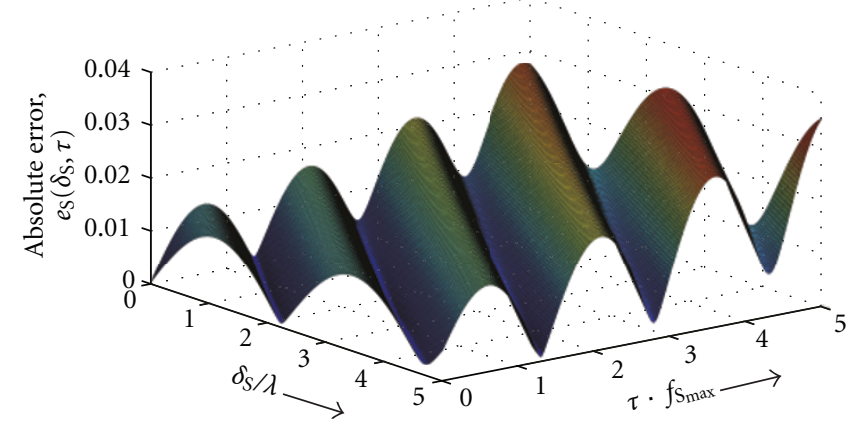

Figure 11: Absolute error $e_{\mathrm{S}}\left(\delta_{\mathrm{S}}, \tau\right)=\left|\rho_{\mathrm{S}}\left(\delta_{\mathrm{S}}, \tau\right)-\hat{\rho}_{\mathrm{S}}\left(\delta_{\mathrm{S}}, \tau\right)\right|$ by using the MMEA with $M=40$ (nonisotropic scattering, von Mises density with $\phi_{\mathrm{S}}^{(0)}=60^{\circ}$ and $\left.\kappa_{\mathrm{S}}=40\right)$.

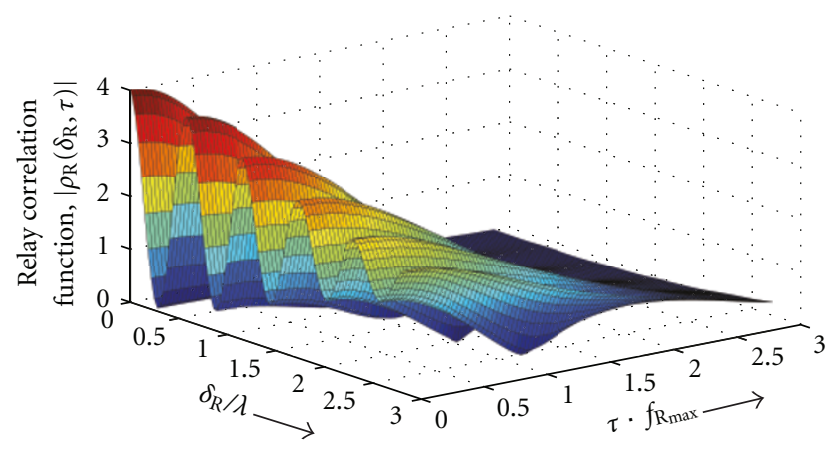

Figure 12: Absolute value of the relay CF $\left|\rho_{\mathrm{R}}\left(\delta_{\mathrm{R}}, \tau\right)\right|$ of the $2 \times$ $2 \times 2$ MIMO M2M reference channel model under nonisotropic scattering conditions (von Mises density with $\phi_{\mathrm{S}-\mathrm{R}}^{(0)}=\phi_{\mathrm{R}-\mathrm{D}}^{(0)}=60^{\circ}$ and $\left.\kappa_{\mathrm{S}-\mathrm{R}}=\kappa_{\mathrm{R}-\mathrm{D}}=40\right)$.

for computing the simulation model parameters when the AODs are non-uniformly distributed on the rings around the source mobile station [30]. The successful application of the LPNM for minimizing the error function of the one-ring model parameters and the two-ring model parameters can be found in [19] and [20], respectively. It is, therefore, believed that the LPNM is equally beneficial for the computation of the three-ring model parameters under nonisotropic scattering conditions. The absolute value of the reference model's relay CF $\left|\rho_{\mathrm{R}}\left(\delta_{\mathrm{R}}, \tau\right)\right|$ (see (38)) and the absolute value of the simulation model's source CF $\left|\hat{\rho}_{\mathrm{R}}\left(\delta_{\mathrm{R}}, \tau\right)\right|$ (see (31)) are shown in Figures 12 and 13, respectively. The measure of the quality of the approximation $\rho_{\mathrm{R}}\left(\delta_{\mathrm{R}}, \tau\right) \approx$ $\hat{\rho}_{\mathrm{R}}\left(\delta_{\mathrm{R}}, \tau\right)$, that is, the absolute error $e_{\mathrm{R}}\left(\delta_{\mathrm{R}}, \tau\right)$ is illustrated in Figure 14. It can be seen in Figure 14 that the maximum value of $e_{\mathrm{R}}\left(\delta_{\mathrm{R}}, \tau\right)$ is less than $10^{-1}$. The same arguments given for minimizing $e_{S}\left(\delta_{S}, \tau\right)$ by using the LPNM are valid for minimizing $e_{\mathrm{R}}\left(\delta_{\mathrm{R}}, \tau\right)$.

\section{Conclusion}

In this article, we have derived a reference model and a stochastic simulation model for narrowband MIMO $\mathrm{M} 2 \mathrm{M}$ fading channel for relay-based cooperative networks. 


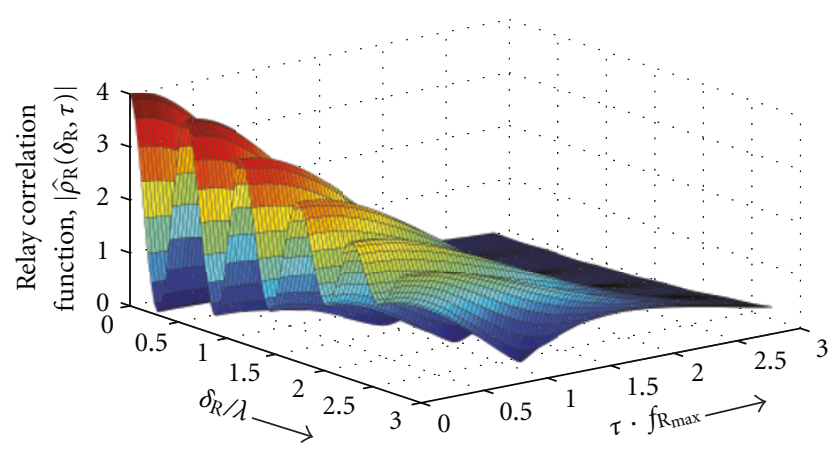

Figure 13: Absolute value of the relay CF $\left|\hat{\rho}_{\mathrm{R}}\left(\delta_{\mathrm{R}}, \tau\right)\right|$ of the $2 \times 2 \times$ 2 MIMO M2M stochastic channel simulator designed by applying the MMEA with $K=L=23$ (nonisotropic scattering, von Mises density with $\phi_{\mathrm{S}-\mathrm{R}}^{(0)}=\phi_{\mathrm{R}-\mathrm{D}}^{(0)}=60^{\circ}$ and $\left.\kappa_{\mathrm{S}-\mathrm{R}}=\kappa_{\mathrm{R}-\mathrm{D}}=40\right)$.

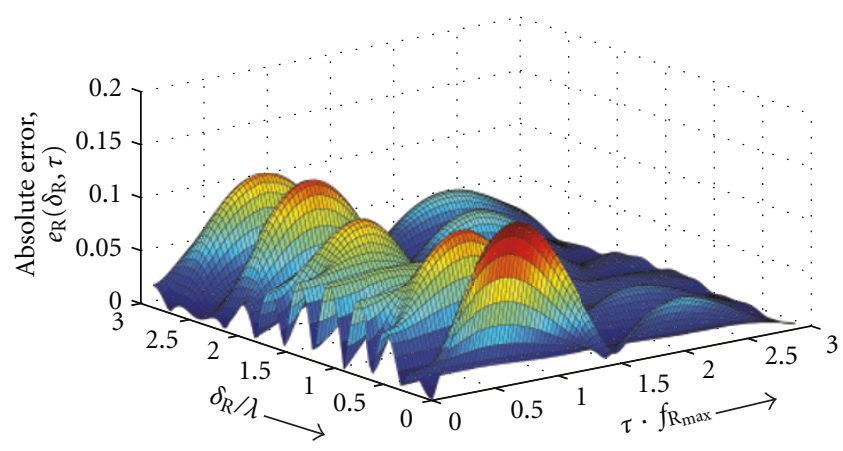

FIGURE 14: Absolute error $e_{\mathrm{R}}\left(\delta_{\mathrm{R}}, \tau\right)=\left|\rho_{\mathrm{R}}\left(\delta_{\mathrm{R}}, \tau\right)-\hat{\rho}_{\mathrm{R}}\left(\delta_{\mathrm{R}}, \tau\right)\right|$ by using the MMEA with $K=L=23$ (nonisotropic scattering, von Mises density with $\phi_{S-R}^{(0)}=\phi_{R-D}^{(0)}=60^{\circ}$ and $\left.\kappa_{S-R}=\kappa_{R-D}=40\right)$.

The starting point for deriving the reference model was the geometrical three-ring scattering model, where it is assumed that the local scatterers are located on rings around the source mobile station, the mobile relay, and the destination mobile station. Furthermore, the suggested three-ring scattering model came out to be a concatenation of two separate two-ring scattering models. General analytical formulas along with exact closed-form expressions for the source (destination) CF and the relay CF specific to nonisotropic scattering have been presented. The results show that various CCFs describing the simulation model closely approximate the corresponding CCFs of the reference model. The developed channel simulator is useful for analyzing the dynamic behavior of the MIMO channel capacity of relay-based M2M communication systems. In addition, the proposed geometrical three-ring scattering model for narrowband MIMO M2M fading channels is the groundwork towards the development and analysis of new channels models for wideband MIMO M2M fading channels in cooperative networks.

\section{Acknowledgment}

The material in this paper was presented in part at the IEEE 69th Vehicular Technology Conference, VTC-Spring 2009, Barcelona, Spain, April 2009.

\section{References}

[1] S. Barbarossa and G. Scutari, "Cooperative diversity through virtual arrays in multihop networks," in Proceedings of the IEEE International Conference on Accoustics, Speech, and Signal Processing, pp. 209-212, Hong Kong, April 2003.

[2] A. Sendonaris, E. Erkip, and B. Aazhang, "User cooperation diversity_part I: system description," IEEE Transactions on Communications, vol. 51, no. 11, pp. 1927-1938, 2003.

[3] A. Sendonaris, E. Erkip, and B. Aazhang, "User cooperation diversity_part II: implementation aspects and performance analysis," IEEE Transactions on Communications, vol. 51, no. 11, pp. 1939-1948, 2003.

[4] J. N. Laneman, D. N. C. Tse, and G. W. Wornell, "Cooperative diversity in wireless networks: efficient protocols and outage behavior," IEEE Transactions on Information Theory, vol. 50, no. 12, pp. 3062-3080, 2004.

[5] A. S. Akki and F. Haber, "A statistical model of mobile-tomobile land communication channel," IEEE Transactions on Vehicular Technology, vol. VT-35, no. 1, pp. 2-7, 1986.

[6] A. S. Akki, "Statistical properties of mobile-to-mobile land communication channels," IEEE Transactions on Vehicular Technology, vol. 43, no. 4, pp. 826-831, 1994.

[7] B. Talha and M. Pätzold, "On the statistical properties of double Rice channels," in Proceedings of the 10th International Symposium on Wireless Personal Multimedia Communications (WPMC '07), pp. 517-522, Jaipur, India, December 2007.

[8] B. Talha and M. Pätzold, "Statistical modeling and analysis of mobile-to-mobile fading channels in cooperative networks under line-of-sight conditions," Wireless Personal Communications, vol. 54, no. 1, pp. 3-19, 2010.

[9] B. Talha and M. Pätzold, "Mobile-to-mobile fading channels in amplify-and-forward relay systems under line-ofsight conditions: statistical modeling and analysis," Annals of Telecommunications, vol. 65, no. 7-8, pp. 391-410, 2010.

[10] V. Erceg, S. J. Fortune, J. Ling, A. J. Rustako Jr., and R. A. Valenzuela, "Comparisons of a computer-based propagation prediction tool with experimental data collected in urban microcellular environments," IEEE Journal on Selected Areas in Communications, vol. 15, no. 4, pp. 677-684, 1997.

[11] C. S. Patel, G. L. Stüber, and T. G. Pratt, "Statistical properties of amplify and forward relay fading channels," IEEE Transactions on Vehicular Technology, vol. 55, no. 1, pp. 1-9, 2006.

[12] I. Z. Kovács, P. C. F. Eggers, K. Olesen, and L. G. Petersen, "Investigations of outdoor-to-indoor mobile-to-mobile radio communication channels," in Proceedings of the 56th Vehicular Technology Conference (VTC '02), pp. 430-434, Vancouver, Canada, September 2002.

[13] J. B. Andersen, "Statistical distributions in mobile communications using multiple scattering," in Proceedings of the 27th URSI General Assembly, Maastricht, The Netherlands, August 2002.

[14] J. Salo, H. M. El-Sallabi, and P. Vainikainen, "Statistical analysis of the multiple scattering radio channel," IEEE Transactions on Antennas and Propagation, vol. 54, no. 11, pp. 3114-3124, 2006. 
[15] G. J. Foschini and M. J. Gans, "On limits of wireless communications in a fading environment when using multiple antennas," Wireless Personal Communications, vol. 6, no. 3, pp. 311-335, 1998.

[16] E. Telatar, "Capacity of multi-antenna Gaussian channels," European Transactions on Telecommunications, vol. 10, no. 6, pp. 585-595, 1999.

[17] C. S. Patel, G. L. Stüber, and T. G. Pratt, "Comparative analysis of statistical models for the simulation of Rayleigh faded cellular channels," IEEE Transactions on Communications, vol. 53, no. 6, pp. 1017-1026, 2005.

[18] D. S. Shiu, G. J. Foschini, M. J. Gans, and J. M. Kahn, “Fading correlation and its effect on the capacity of multielement antenna systems," IEEE Transactions on Communications, vol. 48 , no. 3, pp. 502-513, 2000.

[19] M. Pätzold and B. O. Hogstad, "A space-time channel simulator for MIMO channels based on the geometrical onering scattering model," Wireless Communications and Mobile Computing, vol. 4, no. 7, pp. 727-737, 2004.

[20] M. Pätzold and B. O. Hogstad, "Design and performance of MIMO channel simulators derived from the two-ring scattering model," in Proceedings of the 14th IST Mobile \& Communications Summit (IST '05), Dresden, Germany, June 2005, paper no. 121.

[21] G. J. Byers and F. Takawira, "Spatially and temporally correlated MIMO channels: modeling and capacity analysis," IEEE Transactions on Vehicular Technology, vol. 53, no. 3, pp. 634643, 2004.

[22] Z. Tang and A. S. Mohan, "A correlated indoor MIMO channel model," in Proceedings of the Canadian Conference on Electrical and Computer Engineering (CCECE'03), vol. 3, pp. 1889-1892, Venice, Italy, May 2003.

[23] R. B. Ertel, P. Cardieri, K. W. Sowerby, T. S. Rappaport, and J. H. Reed, "Overview of spatial channel models for antenna array communication systems," IEEE Personal Communications, vol. 5, no. 1, pp. 10-22, 1998.

[24] R. B. Ertel and J. H. Reed, "Angle and time of arrival statistics for circular and elliptical scattering models," IEEE Journal on Selected Areas in Communications, vol. 17, no. 11, pp. 18291840, 1999.

[25] M. Pätzold and N. Youssef, "Modelling and simulation of direction-selective and frequency-selective mobile radio channels," AEU-International Journal of Electronics and Communications, vol. 55, no. 6, pp. 433-442, 2001.

[26] A. Abdi and M. Kaveh, "A space-time correlation model for multielement antenna systems in mobile fading channels," IEEE Journal on Selected Areas in Communications, vol. 20, no. 3, pp. 550-560, 2002.

[27] T. A. Chen, M. P. Fitz, W. K. Kuo, M. D. Zoltowski, and J. H. Grimm, "Space-time model for frequency nonselective Rayleigh fading channels with applications to space-time modems," IEEE Journal on Selected Areas in Communications, vol. 18, no. 7, pp. 1175-1190, 2000.

[28] Y. Ma and M. Pätzold, "A wideband one-ring MIMO channel model under non-isotropic scattering conditions," in Proceedings of the IEEE Vehicular Technology Conference (VTC'08), pp. 424-429, Marina Bay, Singapore, May 2008.

[29] M. Pätzold and B. O. Hogstad, "A wideband MIMO channel model derived from the geometric elliptical scattering model," Wireless Communications and Mobile Computing, vol. 8, no. 5, pp. 597-605, 2008.
[30] M. Pätzold, B. O. Hogstad, and N. Youssef, "Modeling, analysis, and simulation of MIMO mobile-to-mobile fading channels," IEEE Transactions on Wireless Communications, vol. 7, no. 2, pp. 510-520, 2008.

[31] A. G. Zajić and G. L. Stüber, "Space-time correlated mobileto-mobile channels: modelling and simulation," IEEE Transactions on Vehicular Technology, vol. 57, no. 2, pp. 715-726, 2008.

[32] A. Chelli and M. Pätzold, "A MIMO mobile-to-mobile channel model derived from a geometric street scattering model," in Proceedings of the 4th IEEE International Symposium on Wireless Communication Systems 2007 (ISWCS '07), pp. 792-797, Trondheim, Norway, October 2007.

[33] A. Chelli and M. Pätzold, "A non-stationary MIMO vehicleto-vehicle channel model based on the geometrical T-junction model," in Proceedings of the International Conference on Wireless Communications and Signal Processing (WCSP '09), Nanjing, China, November 2009.

[34] A. G. Zajić and G. L. Stüber, "Three-dimensional modeling, simulation, and capacity analysis of space-time correlated mobile-to-mobile channels," IEEE Transactions on Vehicular Technology, vol. 57, no. 4, pp. 2042-2054, 2008.

[35] K. V. Mardia and P. E. Jupp, Directional Statistics, Chichester: John Wiley \& Sons, 1999.

[36] A. Abdi, J. A. Barger, and M. Kaveh, "A parametric model for the distribution of the angle of arrival and the associated correlation function and power spectrum at the mobile station," IEEE Transactions on Vehicular Technology, vol. 51, no. 3, pp. 425-434, 2002.

[37] I. S. Gradshteyn and I. M. Ryzhik, Table of Integrals, Series, and Products, Academic Press, New York, NY, USA, 6th edition, 2000.

[38] B. Talha and M. Pätzold, "A geometrical channel model for MIMO mobile-to-mobile fading channels in cooperative networks," in Proceedings of the 69th IEEE Vehicular Technology Conference (VTC '09), Barcelona, Spain, April 2009.

[39] C. A. Gutierrez-Diaz-de-Leon and M. Pätzold, "Sum-ofsinusoids-based simulation of flat fading wireless propagation channels under non-isotropic scattering conditions," in Proceedings of the 50th Annual IEEE Global Telecommunications Conference (GLOBECOM '07), pp. 3842-3846, Washington, DC, USA, November 2007.

[40] M. Pätzold, Mobile Fading Channels, John Wiley \& Sons, Chichester, UK, 2002. 